\title{
Diatomáceas (Bacillariophyta) marinhas e estuarinas do Paraná, Sul do Brasil: lista de espécies com ênfase em espécies nocivas
}

\author{
Leticia Knechtel Procopiak ${ }^{1}$ Luciano Felício Fernandes², Hermes Moreira-Filho²
}

Biota Neotropica v6 (n3) -http://www.biotaneotropica.org.br/v6n3/pt/abstract?inventory+bn02306032006

\author{
Recebido em 08/03/06. \\ Versão reformulada recebida em 04/09/06 \\ Publicado em 11/10/06
}

\begin{abstract}
${ }^{1}$ Associação de Defesa do Meio Ambiente e do Desenvolvimento de Antonina (ADEMADAN) - Praça Coronel Macedo, s/n - Centro - CEP 83.370-000, Antonina, Paraná (http://www.ademadan.org.br)

${ }^{1}$ Universidade Federal do Paraná - Doutoranda em Meio Ambiente e Desenvolvimento - Rua dos Funcionários, 1540 Juvevê-CEP 80.035-050, Curitiba, Paraná.

${ }^{2}$ Universidade Federal do Paraná - Setor de Ciências Biológicas - Departamento de Botânica - Caixa Postal 19031

CEP 81531-990, Curitiba, Paraná (http://www.ufpr.br/)

Correspondence to: Leticia Knechtel Procopiak (mailto:leprocopiak@yahoo.com.br)
\end{abstract}

\begin{abstract}
Procopiak, L.K., Fernandes, L.F. and Moreira Filho, H. Marine and estuarine diatoms (Bacillariophyta) from Parana, southern Brazil: check-list with emphasis on harmful species. Biota Neotrop. Sep/Dec 2006 vol. 6, no. 3 http:// www.biotaneotropica.org.br/v6n3/pt/abstract?inventory+bn02306032006 ISSN 1676-0603
\end{abstract}

The species check-lists are important to know the local biodiversity. If they are harmful and/or exotic they can cause environmental damages. The portuary regions' aquatic biota survey is valuable to inform which are the high risk species in the water (exotic or harmful species). The species can be caught when the ships are anchored in Parana harbors during the ballast taken. After that, these species can be transferred to the next harbor where it will be discharged, so it starts the biological invasion. In this article the marine and estuarine diatoms of Parana have been listed based on some issues that have been published since 1918 and over the results of ALARME Project (Ballast Water: Risk analysis, Environmental Management Plan and Monitoring of Exotic Species in Paranagua Harbor, Parana) in the Estuarine Paranagua Complex. An amount of 789 specific taxa was recorded and distributed in 167 genera on Parana shore. Among the 789 a number of 575 specific taxa is distributed in 152 genera which were recorded in the Estuarine Paranagua Complex. Some of these species are considered potentially harmful for their toxins production i. e. Amphora coffaeformis and Pseudo-nitzschia spp. The other ones have harmful effects because of anoxia due to the excess of biomass decomposed by bacteriae during the blooming periods. Furthermore it can happens excessive oxygen consumption by algae during breathing. These species are Asterionellopsis glaciallis, Cerataulina pelagica, Coscinodiscus spp, Cylindrotheca closterium, Leptocylindrus spp and Skeletonema costatum. Furthermore, Chaetoceros spp, have setae that damage the fishes' gills causing mass mortalities in some regions. Some species found in samples of the ALARME project are new records for the Parana coast such as Bellerochea horologicales, Biremis circumtexta, Gyrosigma macrum, Licmophora remulus, Nitzschia behrei, Pseudonitzschia calliantha, P. multisseries and Thalassiosira subtilis var. máxima.

Key words: harmful diatoms, check-list, ballast water, Parana.

http://www.biotaneotropica.org.br 


\section{Resumo}

Procopiak, L.K., Fernandes, L.F. and Moreira Filho, H. Diatomáceas (Bacillariophyta) marinhas e estuarinas do Paraná, Sul do Brasil: lista de espécies com ênfase em espécies nocivas. Biota Neotrop. Sep/Dec 2006 vol. 6, no. 3 http:// www.biotaneotropica.org.br/v6n3/pt/abstract?inventory+bn02306032006 ISSN 1676-0603

As listagens de espécies são importantes para conhecer a biodiversidade local. Se as espécies são nocivas e/ou exóticas, elas podem causar danos ambientais. O levantamento da biota aquática de regiões portuárias é valioso para informar quais as espécies de alto risco estão presentes na água (espécies exóticas e/ou nocivas). As espécies podem ser capturadas quando os navios estão atracados nos portos do Paraná durante a tomada de lastro. Então, estas espécies podem ser transferidas para o próximo porto onde será realizado o deslastro, iniciando-se o processo de invasão biológica. Neste artigo as diatomáceas marinhas e estuarinas do Paraná foram listadas com base em trabalhos publicados desde 1918 e nos resultados do projeto ALARME (Água de Lastro: Análise de Risco, Plano de Manejo Ambiental e Monitoramento de Espécies Exóticas no Porto de Paranaguá, Paraná) no Complexo Estuarino de Paranaguá. Um total de 789 táxons específicos foram registrados, distribuídos em 167 gêneros. Destes, 575 táxons específicos, distribuídos em 152 gêneros, foram registrados para o Complexo Estuarino de Paranaguá. Algumas destas espécies são consideradas potencialmente nocivas, por sua produção de toxinas, como Amphora coffaeformis e Pseudo-nitzschia spp. Outras possuem efeitos nocivos por causarem anoxia devido ao excesso de biomassa que é decomposta por bactérias em períodos de florações. Além disso, pode ocorrer consumo excessivo de oxigênio pelas algas durante a respiração. Estas espécies são Asterionellopsis glacialis, Cerataulina pelagica, Coscinodiscus spp., Cylindrotheca closterium, Leptocylindrus spp. e Skeletonema costatum. Além destas, Chaetoceros spp. possuem setas que danificam as brânquias de peixes, causando massiva mortandade destes em algumas regiões. Algumas espécies encontradas nas amostras do projeto ALARME são novos registros para a costa do Paraná como Bellerochea horologicales, Biremis circumtexta, Gyrosigma macrum, Licmophora remulus, Nitzschia behrei Pseudonitzschia calliantha, P. multisseries e Thalassiosira subtilis var. máxima.

Palavras-chave: diatomáceas nocivas, listagem, água de lastro, Paraná. 


\section{Introdução}

As diatomáceas constituem um dos principais grupos do fitoplâncton em águas marinhas neríticas e estuarinas do Paraná (Brandini \& Fernandes 1996). Geralmente compreendem grande parte da biomassa do fitoplâncton, evidenciando seu papel na teia trófica pelágica e nos fluxos de carbono na região de estudo (Brandini et al. 2001). Apesar disso, poucos trabalhos em ecologia citam as espécies dominantes, registrando apenas os gêneros aos quais pertencem. Por outro lado, a taxonomia das diatomáceas é bem estudada no Paraná, representando uma exceção quando comparada com outras regiões do Brasil.

As listagens de espécies são importantes para conhecer a biodiversidade local, principalmente quando se trata de espécies nocivas e/ou exóticas que possam causar danos ao ambiente que impactam. Existem programas internacionais que coordenam uma série de trabalhos, envolvendo também as microalgas, como o Programa HAB (Harmful Algal Blooms), para espécies nocivas que causam florações, iniciado pelos membros da Comissão Oceanográfica Intergovernamental (COI) da UNESCO (International Oceanographic Commission 2005). Recentemente, a preocupação com a invasão de espécies exóticas e potencialmente nocivas via água de lastro de navios intensificou os levantamentos de biota em regiões próximas a portos, a fim de detectar a presença de espécies exóticas e/ou potencialmente nocivas que possam ocorrer nas águas que os cercam e, consequentemente mitigar os possíveis danos causados por elas. Para coordenar as atividades voltadas às invasões biológicas por água de lastro de navios, foi criado o programa internacional GLOBALLAST (Global Ballast Water Management Program) (Global Ballast Water Management Program 2006).

No Paraná está sendo desenvolvido o projeto intitulado “Água de Lastro: Análise de Risco, Plano de Manejo Ambiental e Monitoramento de Espécies Exóticas no Porto de Paranaguá, Paraná - ALARME", do qual este trabalho faz parte. Este projeto visa levantar a biota aquática do Complexo Estuarino de Paranaguá, onde o Porto de Paranaguá está situado, com finalidade de detectar as espécies exóticas e/ou potencialmente nocivas que já ocorrem no local.

O levantamento da biota aquática de regiões portuárias é valioso para informar quais as espécies de alto risco estão presentes nestas águas, ou seja, as espécies exóticas e/ou potencialmente nocivas que podem ser captadas durante a tomada de lastro de navios atracados nos portos do Paraná e, que posteriormente podem ser transferidas para o próximo porto, no qual será realizado o deslastro, iniciando-se o processo de invasão biológica.

Assim, este trabalho objetiva caracterizar o litoral do Paraná e o Complexo Estuarino de Paranaguá (CEP) em relação à composição específica das diatomáceas encontradas na literatura, bem como nas amostragens do projeto ALARME.

\section{Material e Métodos}

O litoral paranaense apresenta a extensão de aproximadamente $100 \mathrm{~km}$ entre as coordenadas $25^{\circ} 20^{\prime}$ a 25 '55' S e 48 10' a 48 35' W (Maritns et al. 2004) (Figura 1).

A revisão de literatura abrangeu os trabalhos publicados de 1918 até 2005 .

As amostragens foram realizadas na baía de Paranaguá (Figura 1), através de arrasto vertical do fundo até a superfície com rede de plâncton $(60 \mathrm{~cm}$ X 1,30 m, $20 \mu \mathrm{m}$ de abertura de malha), acondicionadas em frascos de polietileno de $500 \mathrm{ml}$ e preservadas em solução de formaldeído até a concentração final de 1\%. Amostras coletadas com garrafa de Van Dorn (na superfície, na base da zona fótica e no fundo) também foram analisadas, para a observação das células que são facilmente perdidas durante o arrasto da rede de plâncton, devido ao seu reduzido tamanho. No total foram analisadas 38 amostras de fitoplâncton de rede e 109 amostras de fitoplâncton total. As estações de coleta foram as seguintes: E1: "Bóia 12" em frente ao canal da Galheta (25'33'38,2" S, 48'20'31,80" W), E2: Rio do Maciel (253' 41,52" S, 48'25'29,10" W), E3: Porto de Paranaguá (2529’03,0" S, 48³3'02,28" W), E4: Rio Itiberê (2530'55,74" S, 48²9'53,10" W), E5: Europinha (25'28'22,08" S, 48 $\left.38^{\prime} 01,56^{\prime \prime} \mathrm{W}\right)$, E6: Antonina $\left(25^{\circ} 25^{\prime} 10,74^{\prime \prime} \mathrm{S}\right.$, $\left.48^{\circ} 42^{\prime} 14,46^{\prime \prime} \mathrm{W}\right)$.

A preparação para microscopias óptica e eletrônica foi feita de acordo com Hasle \& Fryxell (1970). As lâminas permanentes foram montadas com resina Naphrax (Northern Biological Supplies, $\mathrm{r}=1,74$ ) e analisadas em microscópio óptico Olympus BX30 equipado com contraste de fase e de interferência. Para a análise em microscopia eletrônica de varredura (MEV) a amostra oxidada é depositada em suportes ("stubs") de alumínio e são colocadas para secar naturalmente. Quando completamente secos, os suportes contendo as amostras são cobertos com ouro. Para a análise em microscopia eletrônica de transmissão (MET) a amostra oxidada é despejada em uma pequena grade ("grid") e deixada para secar ao ar livre.

Alguns táxons necessitaram de microscopia eletrônica para a correta identificação como é o caso de Pseudo-nitzschia spp. Para análises em microscopia eletrônica foram utilizados microscópios eletrônicos de varredura Phillips XL30 e de transmissão JEOL JM1200 EXII.

As atualizações das sinonímias foram realizadas com base em trabalhos recentes (Hasle \& Syvertsen 1996, Round \& Bukhtiyarova 1996, Sala et al. 1998, Tavares \& ValenteMoreira 2000, Fernandes \& Souza-Mosimann 2001, Danielidis \& Mann 2002, Håkanson 2002, Lundholm et al. 2003, entre outros), e na discussão com demais especialistas em diatomáceas. 


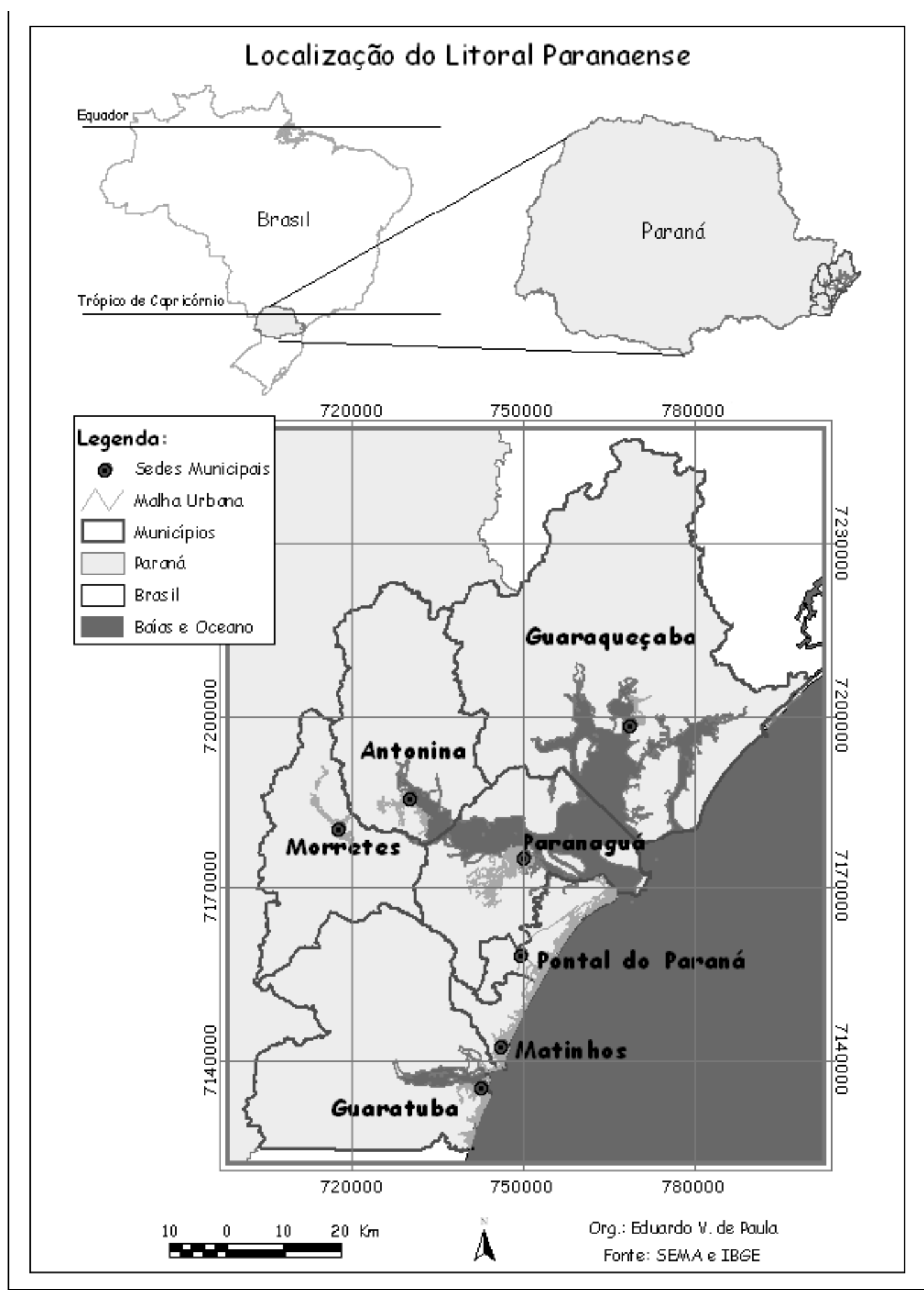

Figura 1 - Mapa mostrando a localização do litoral paranaense. Figure 1 - Map showing the shore of Parana State. 


\section{Resultados e Discussão}

Os trabalhos prévios sobre taxonomia de diatomáceas marinhas e estuarinas no Paraná iniciaram com o trabalho de Cunha \& Fonseca (1918). Neste estudo, realizado no litoral dos Estados do Paraná, Rio Grande do Sul e Santa Catarina, os autores encontraram 17 gêneros de diatomáceas, com 25 espécies. A amostragem no Paraná compreendeu as proximidades da Foz do Rio Itiberê e Baía de Paranaguá. A partir de Moreira-Filho (1959), através da análise de diatomáceas epífitas em Sargassum cymosum C. Agardh var. stenophylum (Metr.) Grunow, coletadas em costão rochoso na Ilha do Farol (praia de Caiobá) deu-se início a um período de intensas investigações sobre as diatomáceas no litoral do Paraná. Diversos autores realizaram levantamentos taxonômicos de diatomáceas ao longo do litoral paranaense (Moreira-Filho 1960, Moreira-Filho 1961, Moreira-Filho \& Kutner 1962, Moreira-Filho \& Mômoli 1962, Moreira-Filho \& Mômoli 1966, Moreira-Filho 1968, MoreiraFilho et al. 1975, Moreira-Filho et al. 1977, Moreira-Filho \& Valente-Moreira 1979, Moreira-Filho \& Valente-Moreira 1980, Valente-Moreira et al. 1980, Moreira-Filho \& Valente-Moreira 1981, Valente-Moreira \& Moreira-Filho 1981, Valente-Moreira \& Moreira-Filho 1982, Oliveira 1983, Valente-Moreira et al. 1985, Valente-Moreira et al. 1986, Valente-Moreira et al. 1987, Valente-Moreira et al. 1994a, Fernandes et al. 1999). A necessidade de organizar as espécies registradas em listas para facilitar a consulta ocorreu a partir do trabalho de Moreira-Filho \& Valente-Moreira (1984) que elaboraram um catálogo contendo as espécies de diatomáceas marinhas e estuarinas do Paraná publicadas no período de 1918 a 1982. Nesta lista já continham 462 espécies, distribuídas em 96 gêneros. Uma nova lista foi publicada por Moreira-Filho et al. (1990) mostrando uma revisão das diatomáceas que ocorrem no Paraná, Santa Catarina e Rio Grande do Sul. Destas, 634 táxons foram registrados para o Paraná. Notase um aumento de 172 espécies que ainda não tinham sido registradas. Até o trabalho de Valente-Moreira et al. (1994b) os trabalhos publicados apresentavam listagens, descrições com ilustrações em desenhos e microscopia óptica. A partir de 1970 a microscopia eletrônica torna-se importante na descrição e identificação de táxons e passa, então, a ser aplicada para a taxonomia das espécies do litoral do Paraná a partir do trabalho de Souza-Mosimann et al. (1997) que analisaram a morfologia e a taxonomia da diatomácea bêntica Margaritum terebro, coletada na Baía de Paranaguá, Paraná. Posteriormente, Fernandes et al. (2001) realizaram cultivo da diatomácea Coscinodiscus wailesii, introduzida na Baía de Paranaguá, mostrando a variação morfológica das valvas de populações cultivadas e naturais, utilizando a microscopia eletrônica de varredura para elucidar algumas estruturas. Fernandes \& Souza-Mosimann (2001) analisaram duas espécies de Triceratium: T. dubium e T. moreirae, também em microscopia eletrônica, provenientes de material coletado em estuários da Baía de Paranaguá e estuário de Santa Catarina. Fernandes et al. (2002) analisaram morfologicamente em microscopia óptica e eletrônica a diatomácea bêntica Nitzschia martiana. E, recentemente Fernandes (2003) analisou a morfologia de Eupodiscus radiatus e Fryxelliela floridana presentes nas águas do Complexo Estuarino de Paranaguá (CEP).

Os estudos sobre a ecologia do fitoplâncton iniciaram-se na Baía de Paranaguá na década de 80 com os trabalhos de Brandini (1985a) e Brandini (1985b), este último realizou estudos ecológicos na Baía de Paranaguá, verificando a distribuição horizontal e sazonal do fitoplâncton, bem como os parâmetros ambientais (temperatura, salinidade, oxigênio e $\mathrm{pH}$ ), biomassa e fotossíntese do fitoplâncton. A partir deste período a ecologia do fitoplâncton passou a ser estudada intensamente por diversos autores (Brandini \& Moraes 1986, Brandini 1988a, b, Brandini et al. 1989, Brandini 1990 a, b, Brandini \& Thamm 1994, Brandini \& Fernandes 1996, Brandini et al. 1997 e Brandini et al. 2001). Brandini \& Fernandes (1996) mostraram que a comunidade fitoplanctônica da plataforma do Paraná é formada por associação costeira, composta por espécies neríticas eurihalinas (Skeletonema costatum, Chaetoceros sp.) e diatomáceas bênticas ressuspendidas. Posteriormente, Fernandes \& Brandini (2004) analisaram a variação sazonal das diatomáceas em águas neríticas do Paraná, a fim de detectar os fatores ambientais que interferem na comunidade fitoplanctônica. Estes autores verificaram que as espécies dominantes foram Cerataulina pelagica, Chaetoceros spp., Dactyliosolen fragilissimus, Guinardia delicatula, Lauderia annulata, Leptocylindrus spp., Pseudo-nitzschia delicatissima, P. australis, Rhizosolenia spp., Skeletonema costatum e Thalassionema nitzschioides.

Com base nos trabalhos revisados desde 1918, foi elaborada uma lista das espécies para o litoral do Paraná (Tabela 1), acrescida das espécies encontradas no projeto ALARME. Foram registradas para o Paraná 789 táxons infragenéricos, distribuídos em 167 gêneros. No Complexo Estuarino de Paranaguá ocorreram 575 táxons infragenéricos, distribuídos em 152 gêneros. Além disso, uma tabela contendo as espécies potencialmente nocivas para o litoral do Paraná foi elaborada com base nos trabalhos levantados e amostras analisadas no projeto ALARME (Tabela 2).

Comparando as espécies registradas neste levantamento com a revisão bibliográfica, constatou-se a presença de alguns táxons que não haviam sido citados nestes trabalhos. São eles: Bellerochea horologicales, Biremis circumtexta, Gyrosigma macrum, Licmophora remulus, Nitzschia behrei, Pseudo-nitzschia calliantha, $P$. multiseries, Thalassiosira subtilis var. maxima.

As espécies potencialmente tóxicas registradas para o litoral do Paraná foram: Amphora coffaeformis (Agardh) Kützing, citada por Valente-Moreira et al. (1994a); Pseudo- 
nitzschia australis Frenguelli, citada por Fernandes \& Brandini (2004); Proença \& Fernandes (2004), Pseudonitzschia delicatissima (Cleve) Heiden, mencionada por Valente-Moreira \& Moreira-Filho (1981); Moreira-Filho \& Valente-Moreira (1984); Brandini (1985a, 1988a); MoreiraFilho et al. (1990); Brandini \& Fernandes (1996); Fernandes \& Brandini (2004) e Proença \& Fernandes (2004), Pseudonitzschia .pungens citada por Moreira-Filho \& ValenteMoreira (1979, 1981, 1984); Valente-Moreira \& Moreira-Filho (1981, 1982); Valente-Moreira et al. (1985); Valente-Moreira et al. (1987); Moreira-Filho et al. (1990); Valente-Moreira et al. (1994a); Fernandes \& Brandini (2004) e Proença \& Fernandes (2004) e Pseudo-nitzschia seriata registrada por Moreira-Filho et al. (1975); Moreira-Filho \& Valente-Moreira (1979, 1984); Valente-Moreira \& Moreira-Filho (1981, 1982); Brandini (1985a); Valente-Moreira et al. (1985); ValenteMoreira et al. (1987); Brandini \& Moraes (1986); Brandini (1988a, b); Moreira-Filho et al. (1990) e Valente-Moreira et al. (1994a); Brandini \& Fernandes (1996). As espécies potencialmente tóxicas Pseudo-nitzschia calliantha e Pseudo-nitzschia multisseries são citadas pela primeira vez neste trabalho. Estas espécies são consideradas potencialmente tóxicas por diversos autores dentre os quais Hasle et al. (1996), Fryxell \& Villac (1999) e Ferrario et al. (2002).

As espécies não tóxicas, mas potencialmente nocivas foram registradas como Asterionellopsis glaciallis citada para o Paraná nos trabalhos de Cunha \& Fonseca (1918); Moreira-Filho (1961); Moreira-Filho et al. (1975, 1990); Moreira-Filho \& Valente-Moreira (1979); Valente-Moreira \& Moreira-Filho (1981, 1982); Moreira-Filho \& Valente-Moreira (1984); Brandini (1985a, b, 1988); Valente-Moreira et al. (1985); Valente-Moreira et al. (1987); Valente-Moreira et al. (1994a, b); Brandini \& Fernandes (1996) e Fernandes \& Brandini (2004). Outra espécie potencialmente nociva e não tóxica é Cerataulina pelagica que foi citada pelos autores Cunha \& Fonseca (1918); Moreira-Filho et al. (1975); Moreira-Filho \& Valente-Moreira (1979, 1984); Valente-Moreira \& MoreiraFilho (1981, 1982); Valente-Moreira et al. (1985); ValenteMoreira et al. (1987); Moreira-Filho et al. (1990); Brandini \& Fernandes (1996) e Fernandes \& Brandini (2004). As espécies do gênero Chaetoceros Ehrenberg, conhecidas na literatura por danificar brânquias de organismos filtradores, foram Chaetoceros concavicornis Manguin mencionada por Valente-Moreira \& Moreira-Filho (1981); Moreira-Filho \& Valente-Moreira (1984); Valente-Moreira et al. (1985); Valente-Moreira et al., (1987); Moreira-Filho et al. (1990); Valente-Moreira et al. (1994a), Chaetoceros convolutus Castracane, citada por Valente-Moreira \& Moreira-Filho (1982); Moreira-Filho \& Valente-Moreira (1984); Brandini (1985a), Valente-Moreira et al. (1985); Valente-Moreira et al. (1994a) e Moreira-Filho et al. (1990) e Chaetoceros danicus Cleve, citada por Brandini (1985a, 1988a); Moreira-Filho et al. (1990) e Fernandes \& Brandini (2004). As espécies de Coscinodiscus Ehrenberg registradas na literatura como potencialmente nocivas e não tóxicas foram: Coscinodiscus centralis Ehrenberg, mencionadas pelos autores ValenteMoreira \& Moreira-Filho (1981); Moreira-Filho \& ValenteMoreira (1984); Brandini (1985a); Valente-Moreira et al. (1985); Valente-Moreira et al. (1987); Moreira-Filho et al. (1990) e Fernandes \& Brandini (2004), Coscinodiscus concinnus Wm. Smith, registrada por Moreira-Filho (1961); Valente-Moreira \& Moreira-Filho (1981, 1982), Moreira-Filho \& Valente-Moreira (1984); Valente-Moreira et al. (1985); Valente-Moreira et al. (1987); Moreira-Filho et al. (1990) e Valente-Moreira et al. (1994b) e Coscinodiscus wailesii, mencionada por Valente-Moreira et al. (1987); Moreira-Filho et al. (1990); Valente-Moreira et al. (1994a); Fernandes et al. (2001); Fernandes \& Brandini (2004) e Proença \& Fernandes (2004). A espécie Cylindrotheca closterium também é considerada potencialmente nociva e não tóxica pela literatura. Para o litoral do Paraná foi registrada pelos autores Moreira-Filho \& Valente-Moreira (1979); Valente-Moreira \& Moreira-Filho (1981, 1982, 1984); Brandini (1985a); ValenteMoreira et al. (1985); Valente-Moreira et al. (1987); MoreiraFilho et al. (1990); Fernandes et al. (1999); Brandini et al. (2001) e Fernandes \& Brandini (2004). Outras espécies do gênero Leptocylindrus Cleve, potencialmente nocivas registradas para o Paraná foram: Leptocylindrus danicus, mencionada por Cunha \& Fonseca (1918); Moreira-Filho et al. (1975); Moreira-Filho \& Valente-Moreira (1979, 1984); Valente-Moreira \& Moreira-Filho (1981, 1982); Brandini (1985a); Valente-Moreira et al. (1985); Valente-Moreira et al. (1987); Brandini \& Moraes (1986); Moreira-Filho et al. (1990) e Fernandes \& Brandini (2004) e Leptocylindrus minimus, registrada por Valente-Moreira \& Moreira-Filho (1981, 1982); Moreira-Filho \& Valente-Moreira (1984); Brandini (1985a, 1985b); Valente-Moreira et al. (1985); Valente-Moreira et al. (1987) e Fernandes \& Brandini (2004). Finalmente, com registros para o Paraná, a espécie potencialmente nociva Skeletonema costatum citada por Cunha \& Fonseca (1918); Moreira-Filho (1961,1975); Moreira-Filho \& Valente-Moreira (1979, 1984), Valente-Moreira \& Moreira-Filho (1981, 1982), Brandini (1985a, 1985b); Valente-Moreira et al. (1985); Brandini \& Moraes (1986); Valente-Moreira et al. (1987); Moreira-Filho et al. (1990); Valente-Moreira et al. (1994a); Valente-Moreira et al. (1994b); Brandini \& Fernandes (1996) e Fernandes \& Brandini (2004). As espécies mencionadas são consideradas potencialmente nocivas, mas não produtoras de toxinas, por diversos autores dentre os quais Fryxell \& Villac (1999); Fernandes et al. (2001); Proença \& Fernandes (2004) e Odebrecht et al. (2002)

Algumas espécies foram consideradas potencialmente nocivas devido à prévia ocorrência de florações relacionadas com a mortandade de organismos filtradores. Como por exemplo, Asterionellopsis glaciallis, no litoral de São Paulo, foi relacionada à mortandade de peixes, mesmo não sendo uma espécie tóxica, mas que devido à combinação de uma série de fatores como ventos fortes, que ressuspendem as células presentes no sedimento, concentrando-as na superfície 
(Odebrecht et al. 2002). A espécie Cerataulina pelagica já causou mortandade em moluscos e peixes, devido à anoxia e ao entupimento das brânquias destes organismos (Hasle \& Fryxell, 1995, Ferrario et al. 2002). As espécies de Chaetoceros citadas possuem setas que em altas concentrações danificam as brânquias de peixes e organismos filtradores, causando sua morte; e Coscinodiscus centralis e C. concinus produzem polissacarídeos que são liberados na água do mar, que em altas concentrações tornam o meio anóxico, causando mortandade dos organismos marinhos e dificultando a migração de peixes (Ferrario et al. 2002). Coscinodiscus wailesii pode produzir grandes quantidades de mucilagem insolúvel que se acumula em organismos planctônicos, aumentadno a densidades da água. Também pode se dividir com muita facilidade o que o torna facilmente adaptável. Esta espécie vem sendo registrada em todo o mundo a partir de 1980 desenvolvendo florações e prejudicando o cultivo de marisco e macroalgas e a pesca comercial, devido à depleção de oxigênio, inclusive para o Complexo Estuarino de Paranaguá (Fernandes et al. 2001, Proença \& Fernandes 2004). Coscinodiscus wailesii esgota os nutrientes da água causando aumento de turbidez da água. No Japão causou necrose em corais e na Itália uma grande quantidade desta espécie produziu muita mucilagem, causando perdas no setor turístico do país (Reguera 2002). A espécie Cylindrotheca closterium já foi relacionada com a produção de agregados mucilagionosos no Mar Adriático, fenômeno conhecido como "Mare sporco" (mar sujo) e afetou o turismo e a pesca (Fanuko et al. 1989, Stachowitsch et al. 1990). A espécie Leptocylindrus danicus já esteve relacionada com a mortandade da pescada (Cynoscion regalis) e duas espécies de salmonídeos (Salmo salar e Oncothynchus kisutch) quando em grandes concentrações (Fryxell \&Villac 1999). Leptocylindrus minimus já esteve associada à morte de salmões em cultivo na costa chilena (Rivera et al. 2002) e da mesma forma que L. danicus, esteve associada à mortandade de pescada e salmonideos (Fryxell \& Villac 1999). A espécie Skeletonema costatum já esteve associada com a mortandade de peixes em cultivos durante períodos de floração no Atlântico Norte (Fryxell \& Villac 1999).

Para a correta identificação de Pseudo-nitzschia spp. foi necessária a utilização de microscopia eletrônica. para observar estruturas indiscerníveis em microscopia óptica para cada espécie. Pseudo-nitzschia calliantha e P. multisseries não haviam sido registradas para o litoral do Paraná, mas sim P. seriata e P. delicatissima. Por outro lado, devido à impossibilidade em diferenciá-las de espécies próximas utilizando-se microscopia óptica, as espécies mencionadas podem corresponder a outros táxons ( $P$. australis e $P$. calliantha, respectivamente). Além disso, a distribuição de $P$. seriata está restrita ao hemisfério norte, indicando sua preferência por águas frias (Hasle et al. 1996 e Hasle 2002). Espécies de Pseudo-nitzschia são produtoras de ácido domóico causando o envenenamento Amnésico por Moluscos (ASP) que é acumulado na cadeia trófica e contamina os organismos aquáticos e os animais que se alimentam delas (Bates et al. 1998; Rines et al. 2002). Os efeitos comuns são distúrbios gastrointestinais, vertigens e nos casos graves, morte (Bates et al. 1989; Fehling et al. 2004).

O litoral do Paraná contém espécies potencialmente nocivas e exóticas que podem prejudicar a pesca e a maricultura durante os períodos de floração, causando prejuízo econômico para as comunidades pesqueiras, uma vez que esta atividade é extremamente importante para a região. $\mathrm{O}$ turismo também pode ser afetado, pois a microalgas excretam compostos protéicos que fornecem cheiro e aspectos desagradáveis na água e, nesta situação, a balneabilidade fica comprometida.

Os portos do Paraná são, principalmente, exportadores de produtos, portanto os navios que chegam para serem carregados estão vazios e com os tanques de lastro cheios de água. Isto torna estes portos grandes importadores de águas de lastro de diversas localidades do Brasil e do exterior, aumentando as possibilidades de invasões biológicas. Os portos do Paraná recebem navios provenientes de países com clima e latitude semelhantes ao litoral paranaense, porém com diatomoflórulas diferentes. Há a possibilidade de espécies que vivem em condições ambientais semelhantes, sobreviverem se forem transportadas pelos navios. Estes navios capturam as espécies de diatomáceas nocivas presentes na região e estas têm chances de se adaptarem em outros portos, se encontrarem condições favoráveis de sobrevivência.

A divulgação da ocorrência de espécies nocivas em regiões portuárias, através de listagens de espécies torna-se imprescindível para auxiliar na elaboração de planos de manejo da água de lastro de navios, a fim de minimizar os impactos ambientais e sócio-econômicos causados por estas espécies.

\section{Agradecimentos}

Trabalho financiado com recursos do projeto ALARME através do Fundo Nacional do Meio Ambiente (FNMA/MMA), contrato CVI 008/2002, sob a coordenação do Prof. Dr. Luciano Felício Fernandes. Os autores agradecem ao Centro de Microscopia Eletrônica da UFPR, ao LACTEC pelas sessões de microscopia eletrônica, à Msc. Priscila Izabel Tremarim e Dr ${ }^{\mathrm{a}}$ Thelma Veiga Ludwig pelo auxílio na nomenclatura de alguns táxons de águas continentais, ao Msc. Eduardo Vedor de Paula pela elaboração do mapa do litoral do Paraná e à Rut Schlichting pelo auxílio na correção do abstract. Parte integrante da Dissertação de Mestrado da primeira autora (bolsista CNPq - 2002 a 2004). 
Procopiak, L.K., Fernandes, L.F. and Moreira Filho, H. - Biota Neotropica, v6 (n3) - bn02306032006

TABELA 1 - Lista das espécies de diatomáceas citadas para o litoral do Paraná, de 1918 a 2005.

*Ocorre no Complexo Estuarino de Paranaguá.

**Primeiro registro de ocorrência no Complexo Estuarino de Paranaguá e litoral paranaense

TABLE 1 - Check-list of diatoms species quoted for the Parana State coast, from 1918 to 2005.

*It occurs in the Estuarine Paranagua Complex.

**First record in the Estuarine Paranagua Complex and Parana coast.

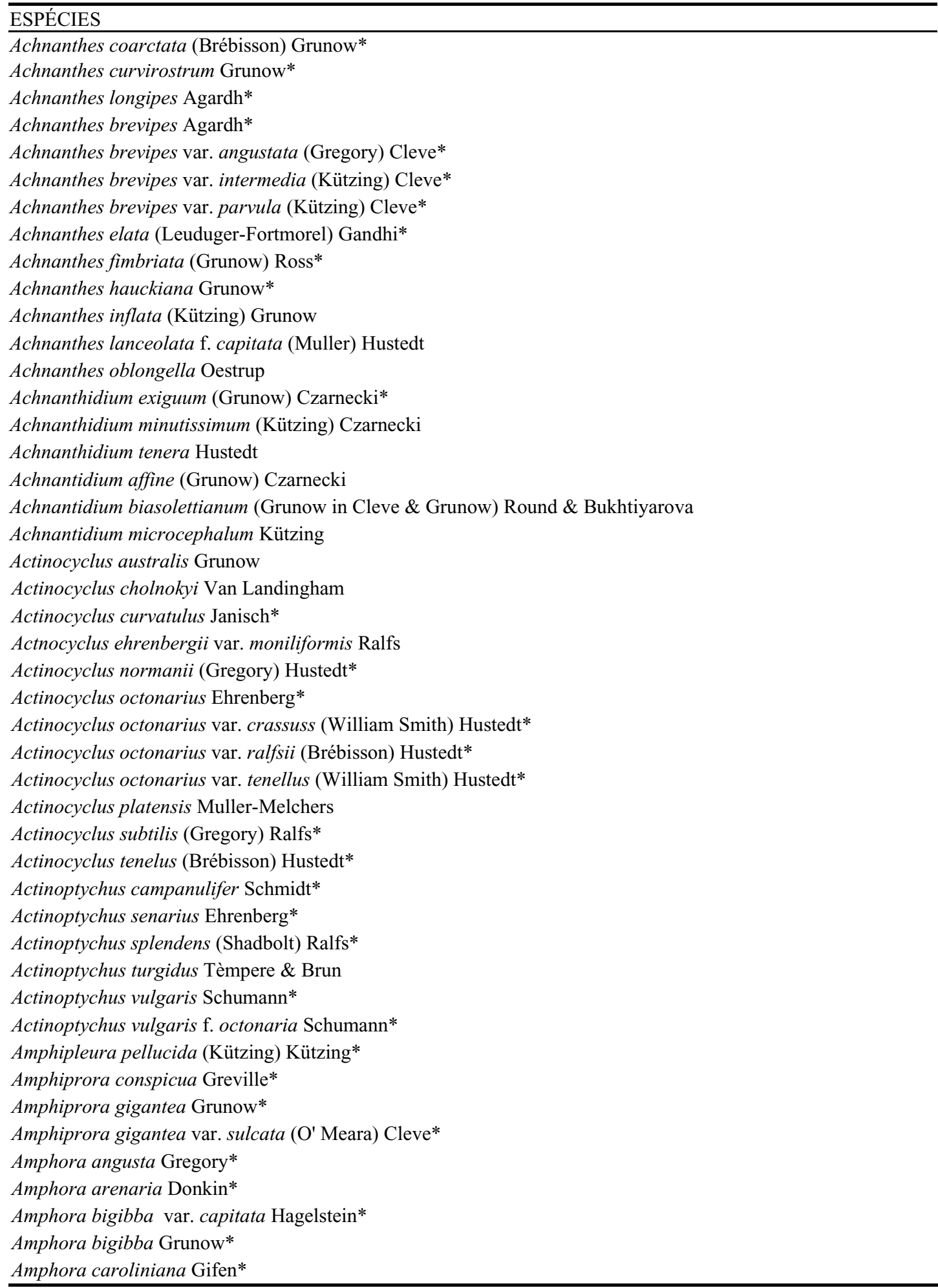

http://www.biotaneotropica.org.br 
TABELA 1 (continuação) - Lista das espécies de diatomáceas citadas para o litoral do Paraná, de 1918 a 2005. TABLE 1 (continuation) - Check-list of diatoms species quoted for the Paraná coast, from 1918 to 2005.

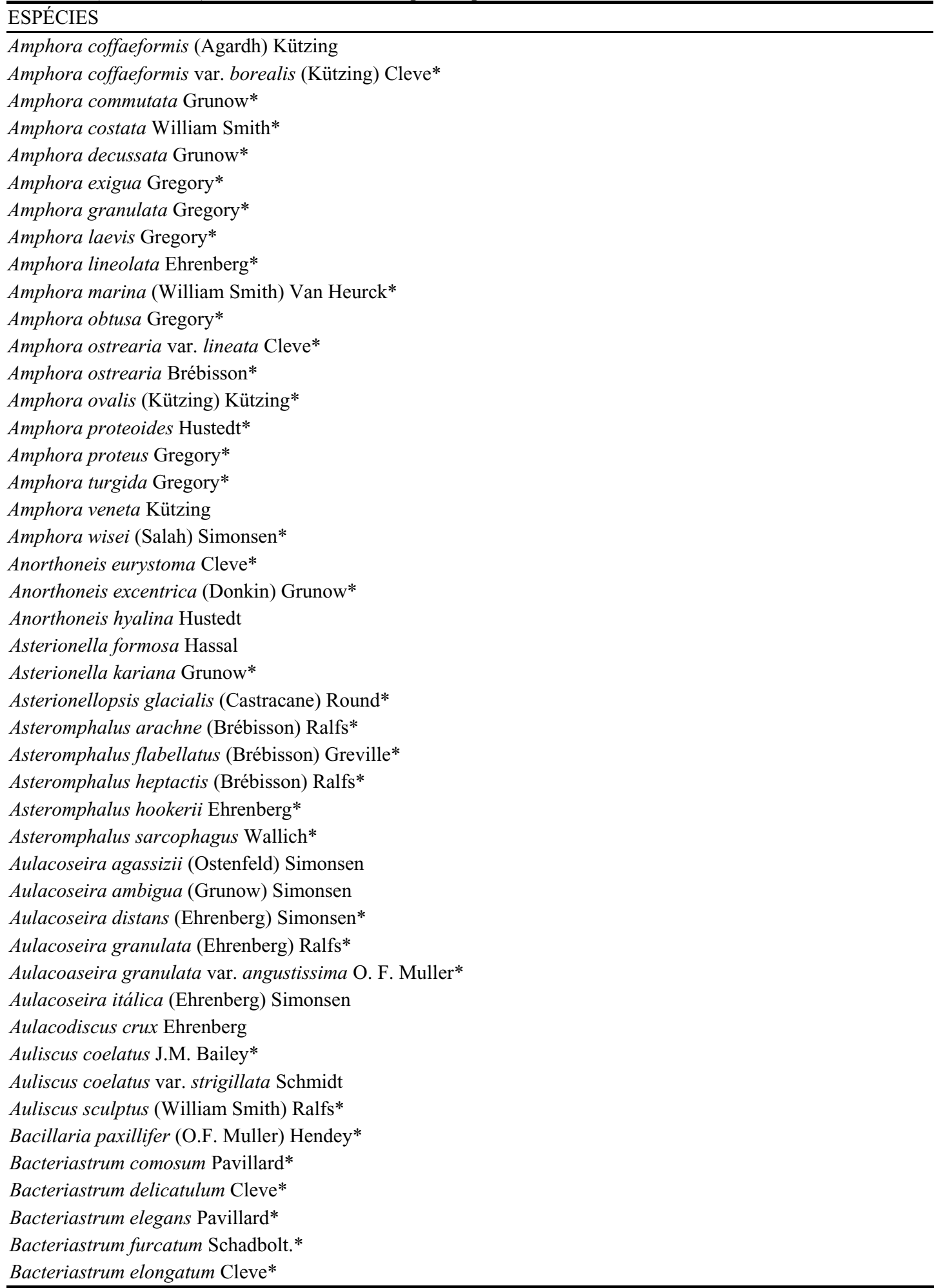

http://www.biotaneotropica.org.br 
TABELA 1 (continuação) - Lista das espécies de diatomáceas citadas para o litoral do Paraná, de 1918 a 2005. TABLE 1 (continuation) - Check-list of diatoms species quoted for the Paraná coast, from 1918 to 2005.

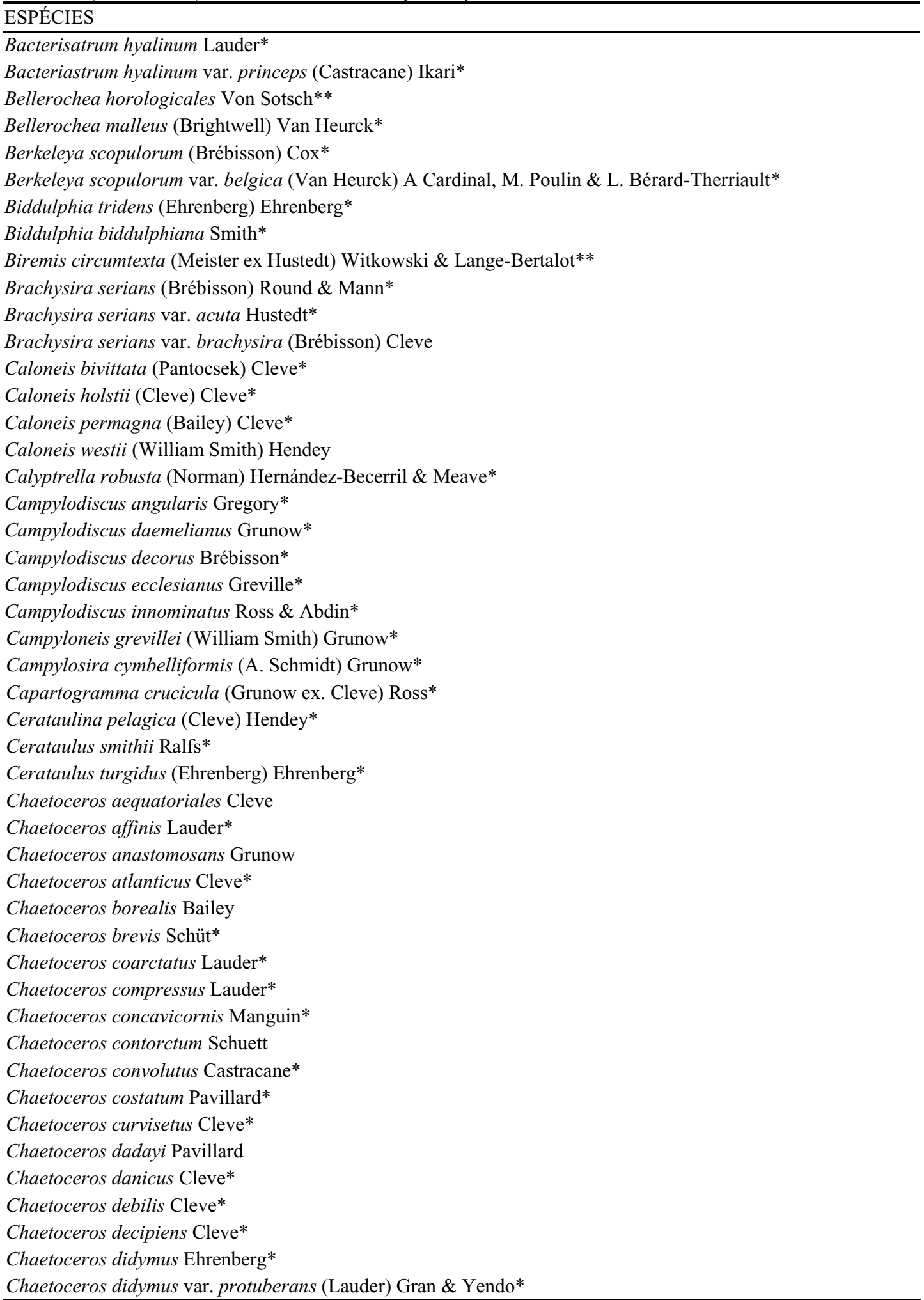

http://www.biotaneotropica.org.br 
TABELA 1 (continuação) - Lista das espécies de diatomáceas citadas para o litoral do Paraná, de 1918 a 2005. TABLE 1 (continuation) - Check-list of diatoms species quoted for the Paraná coast, from 1918 to 2005.

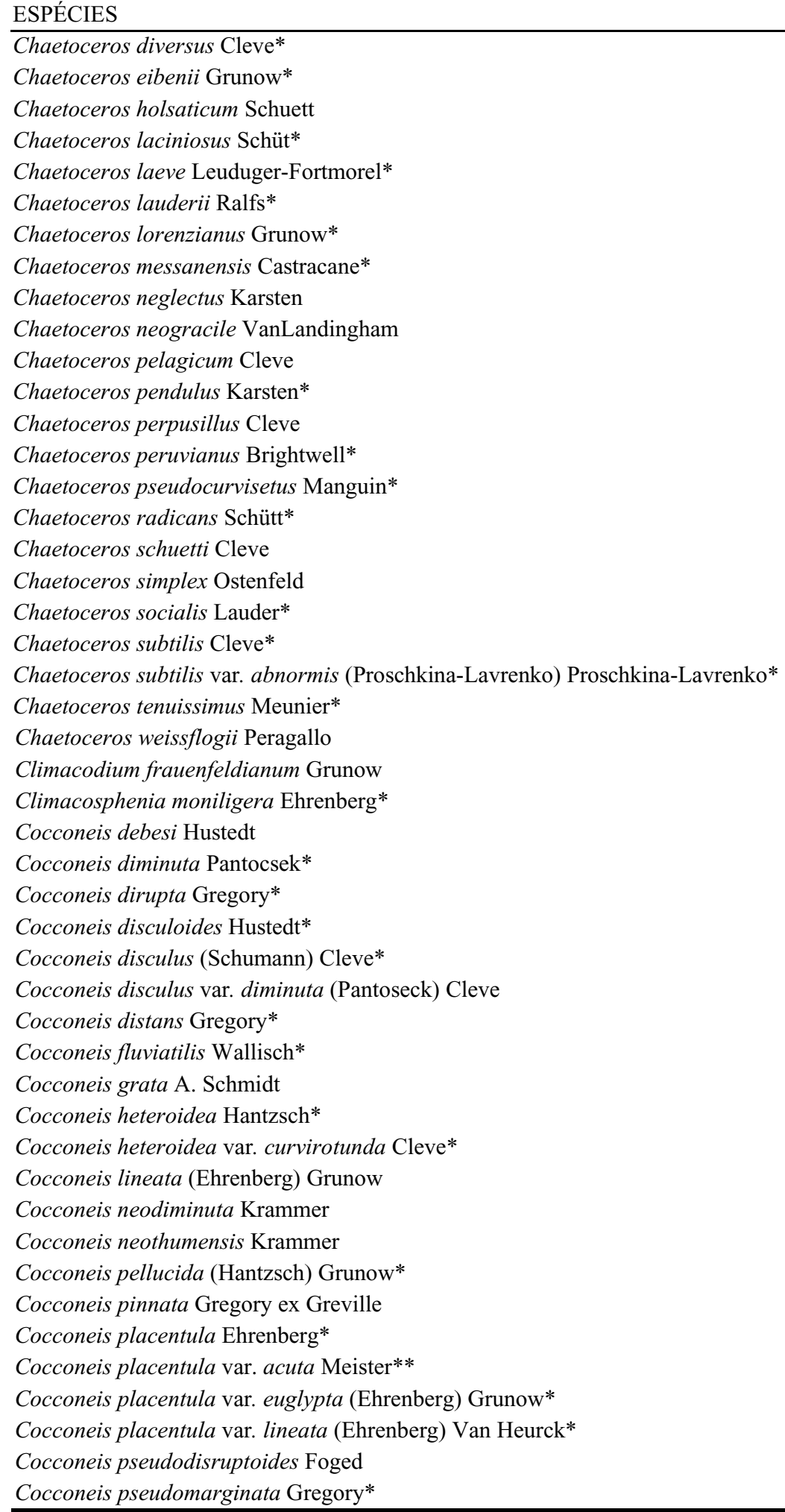

http://www.biotaneotropica.org.br 
TABELA 1 (continuação) - Lista das espécies de diatomáceas citadas para o litoral do Paraná, de 1918 a 2005.

TABLE 1 (continuation) - Check-list of diatoms species quoted for the Paraná coast, from 1918 to 2005.

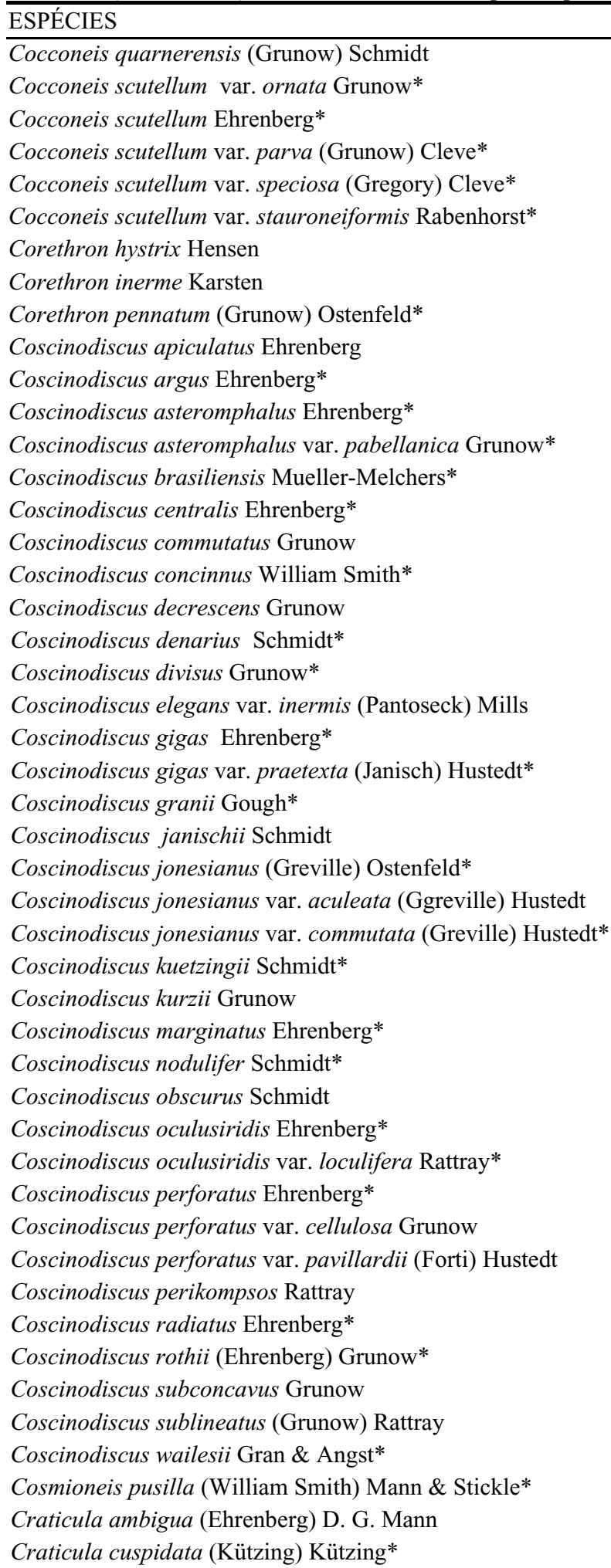

http://www.biotaneotropica.org.br 
TABELA 1 (continuação) - Lista das espécies de diatomáceas citadas para o litoral do Paraná, de 1918 a 2005. TABLE 1 (continuation) - Check-list of diatoms species quoted for the Paraná coast, from 1918 to 2005.

\section{ESPÉCIES}

Craticula halophila (Grunow) Mann

Ctenophora pulchella (Ralfs ex Kutzing) Williams \& Round

Cyclotella comta (Ehrenberg) Kützing*

Cyclotella kuetzingiana Thwaiter*

Cyclotella litoralis Lange \& Syvertsen*

Cyclotella meneghiniana Kützing*

Cyclotella quadrijuncta (Schröer) Hustedt*

Cyclotella striata (Kützing) Grunow*

Cyclotella striata var. ambigua (Grunow) Grunow

Cyclotella stylorum Brightwell*

Cylindrotheca closterium (Ehrenberg) Reim \& Lewin*

Cymatodiscus planetophorus (Meister) Hendey*

Cymatonitzschia marina (Lewis) Simonsen*

Cymatosira belgica Grunow*

Cymatosira lorenziana Grunow*

Cymatosira. atlantica Frenguelli

Cymatotheca weissflogii (Grunow) Hendey*

Cymbella affinis Kützing*

Cymbella amphicephala Naegeli*

Cymbella cymbiformis Agardh*

Cymbella rabenhorstii Ross

Cymbella tumida (Brébisson) Van Heurck

Cymbella turgida Gregory*

Dactyliosolen antarcticus Castracane

Dactyliosolen fragilissimus (Bergon) Hasle*

Dactyliosolen mediterraneus (H. Peragallo) Peragallo

Dactyliosolen tenuijunctus (Manguin) Hasle

Delphineis surirella (Ehrenberg) Andrews*

Delphineis surirella var. australis (Petit) Navarro*

Denticula subtilis Grunow*

Desikaneis gessnerii (Hustedt) Prassad*

Detonula pumila (Castracane) Schutt*

Detonula thermalis Kützing*

Diadesmis contenta (Grunow) Mann*

Dictioneis marginata (Lewis) Cleve*

Dimeregramma marinum (Gregory) Ralfs

Dimeregramma minor (Gregory) Ralfs*

Dimeregramma rostratum Hustedt

Diploneis aestuarii Hustedt*

Diploneis bombus Ehrenberg*

Diploneis cafra (Gifen) Witkowski; Lange-Bertalot \& Metzeltin*

Diploneis crabro (Ehrenberg) Ehrenberg*

Diploneis didyma (Ehrenberg) Ehrenberg*

Diploneis gruendleri (Schmidt) Cleve*

Diploneis incurvata (Gregory) Cleve*

Diploneis interrupta (Kützing) Cleve*

Diploneis oblongella (Naegeli ex. Kützing) Cleve*

http://www.biotaneotropica.org.br 
TABELA 1 (continuação) - Lista das espécies de diatomáceas citadas para o litoral do Paraná, de 1918 a 2005. TABLE 1 (continuation) - Check-list of diatoms species quoted for the Paraná coast, from 1918 to 2005. ESPÉCIES

Diploneis ovalis (Hasle) Cleve*

Diploneis ovalis var. oblongella (Naegeli) Cleve

Diploneis papula (Schmidt) Cleve*

Diploneis papula var. constricta Hustedt*

Diploneis smithii (Brébisson) Cleve*

Diploneis smithii var. rhombica Mereschkowsky

Diploneis subovalis Cleve*

Diploneis vacillans (Schmidt) Cleve*

Diploneis weissflogii (Schmidt) Cleve*

Discostella pseudostelligera (Hustedt) Houk \& Klee

Discostella stelligera (Cleve \& Grunow) Houck \& Klee

Ditylum brightwelli var. pyramidalis H. Ferrando*

Ditylum brightwelli (West) Grunow*

Donkinia carinata (Donkin) Ralfs*

Donkinia recta (Donkin) Grunow*

Encyonema gracile (Ehrenberg) Kützing*

Encyonema lunatum William Smith

Endictya oceanica Ehrenberg

Entomoneis alata Ehrenberg*

Entomoneis alata var. pulchra (Bailey) Cleve*

Epithemia turgida (Ehrenberg) Kützing*

Epithemia zebra (Ehrenberg) Kützing*

Ethmodiscus gazellae (Janisch ex. Grunow) Hustedt*

Eucampia antarctica (Castracane) Mangin

Eucampia cornuta (Cleve) Grunow*

Eucampia zodiacus Ehrenberg*

Eunotia camelus Ehrenberg

Eunotia camelus var. denticulata (Brébisson) Grunow

Eunotia didyma Grunow*

Eunotia flexuosa (Brébisson) Kützing*

Eunotia indica Grunow

Eunotia lineolata Husted*

Eunotia maior (William Smith) Rabenhorst

Eunotia monodon Ehrenberg

Eunotia pectinalis (Dillwin) Rabenhorst*

Eunotia rabenhorstii var. monodon Cleve \& Grunow*

Eunotia sudetica $\mathrm{O}$. Müller*

Eunotia triodon Ehrenberg

Eunotogramma laeve Grunow*

Eunotogramma marinum (William Smith) Peragallo*

Eunotogramma rostratum Hustedt*

Eunotogramma variabilis Grunow

Eupodiscus antiquus (Cox) Hanna*

Eupodiscus radiatus Bailey*

Fallacia forcipata (Greville) Stickle \& Mann*

Fallacia inflata (Heiden) Hustedt*

Fallacia nummularia (Greville) D.G. Mann*

http://www.biotaneotropica.org.br 
TABELA 1 (continuação) - Lista das espécies de diatomáceas citadas para o litoral do Paraná, de 1918 a 2005.

TABLE 1 (continuation) - Check-list of diatoms species quoted for the Paraná coast, from 1918 to 2005.

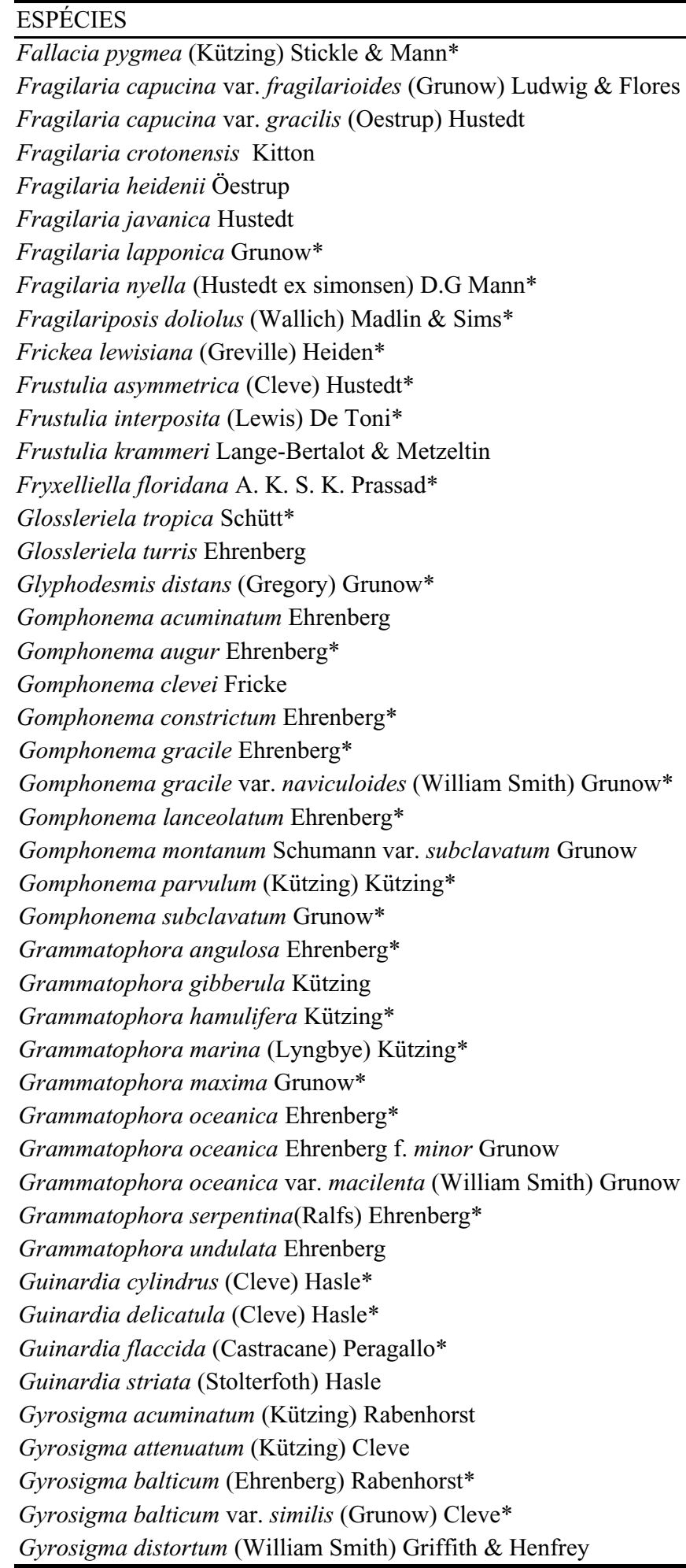

http://www.biotaneotropica.org.br 
TABELA 1 (continuação) - Lista das espécies de diatomáceas citadas para o litoral do Paraná, de 1918 a 2005. TABLE 1 (continuation) - Check-list of diatoms species quoted for the Paraná coast, from 1918 to 2005.

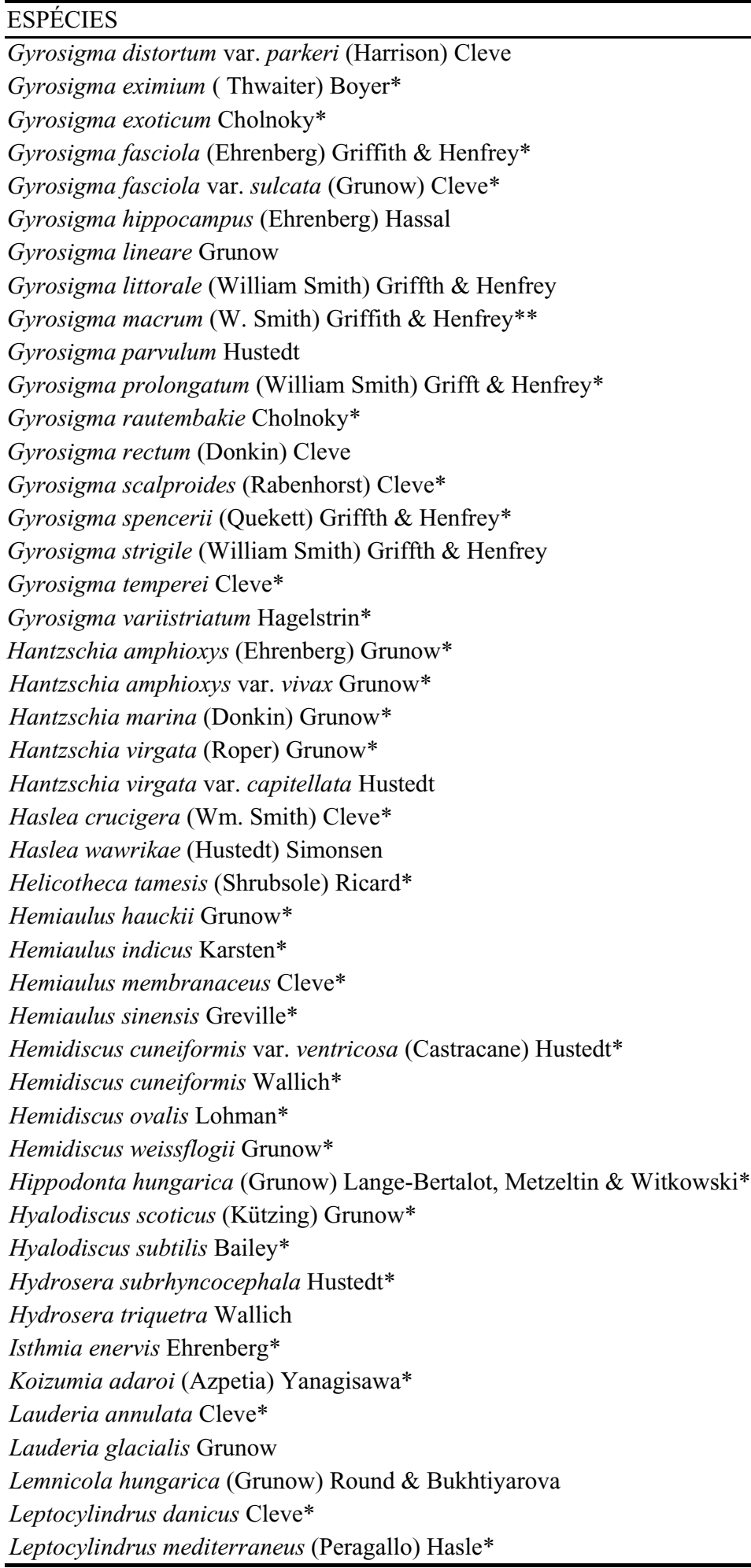

http://www.biotaneotropica.org.br 
TABELA 1 (continuação) - Lista das espécies de diatomáceas citadas para o litoral do Paraná, de 1918 a 2005. TABLE 1 (continuation) - Check-list of diatoms species quoted for the Paraná coast, from 1918 to 2005.

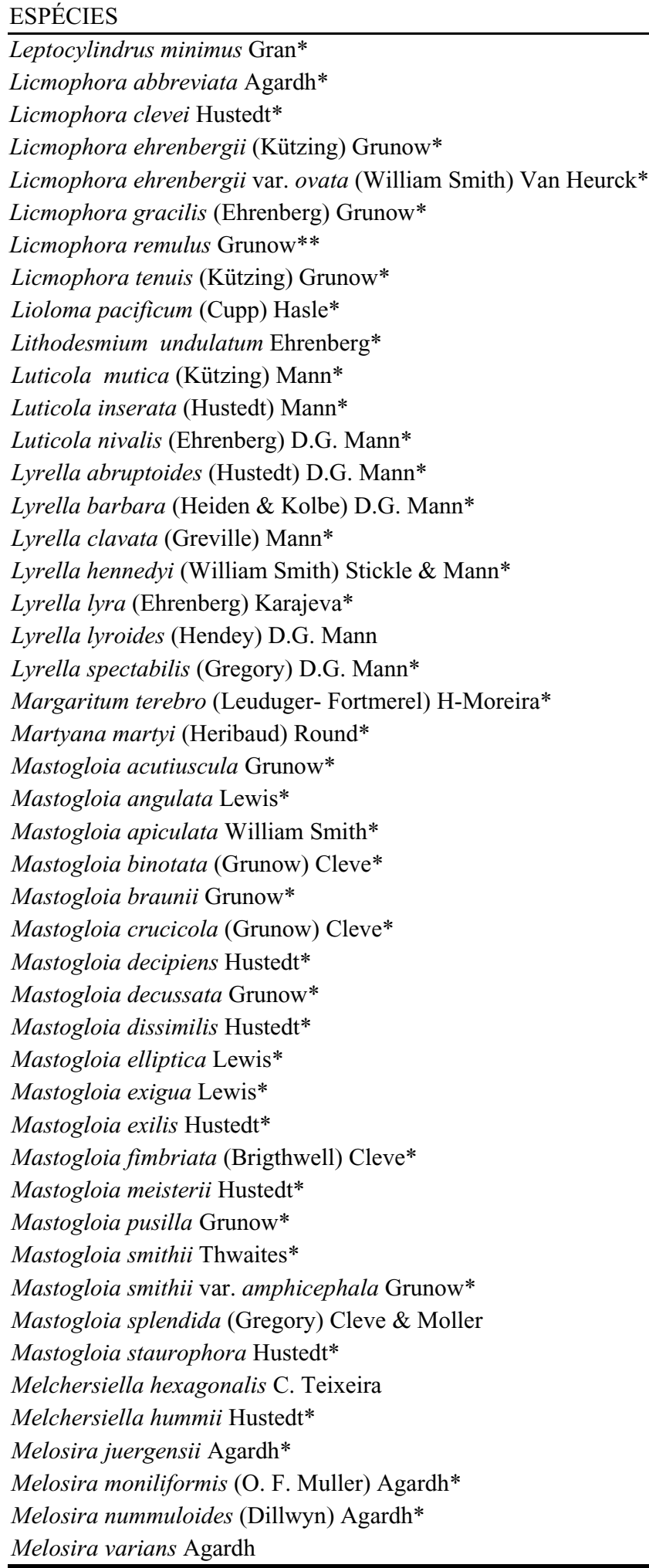

http://www.biotaneotropica.org.br 
TABELA 1 (continuação) - Lista das espécies de diatomáceas citadas para o litoral do Paraná, de 1918 a 2005. TABLE 1 (continuation) - Check-list of diatoms species quoted for the Paraná coast, from 1918 to 2005

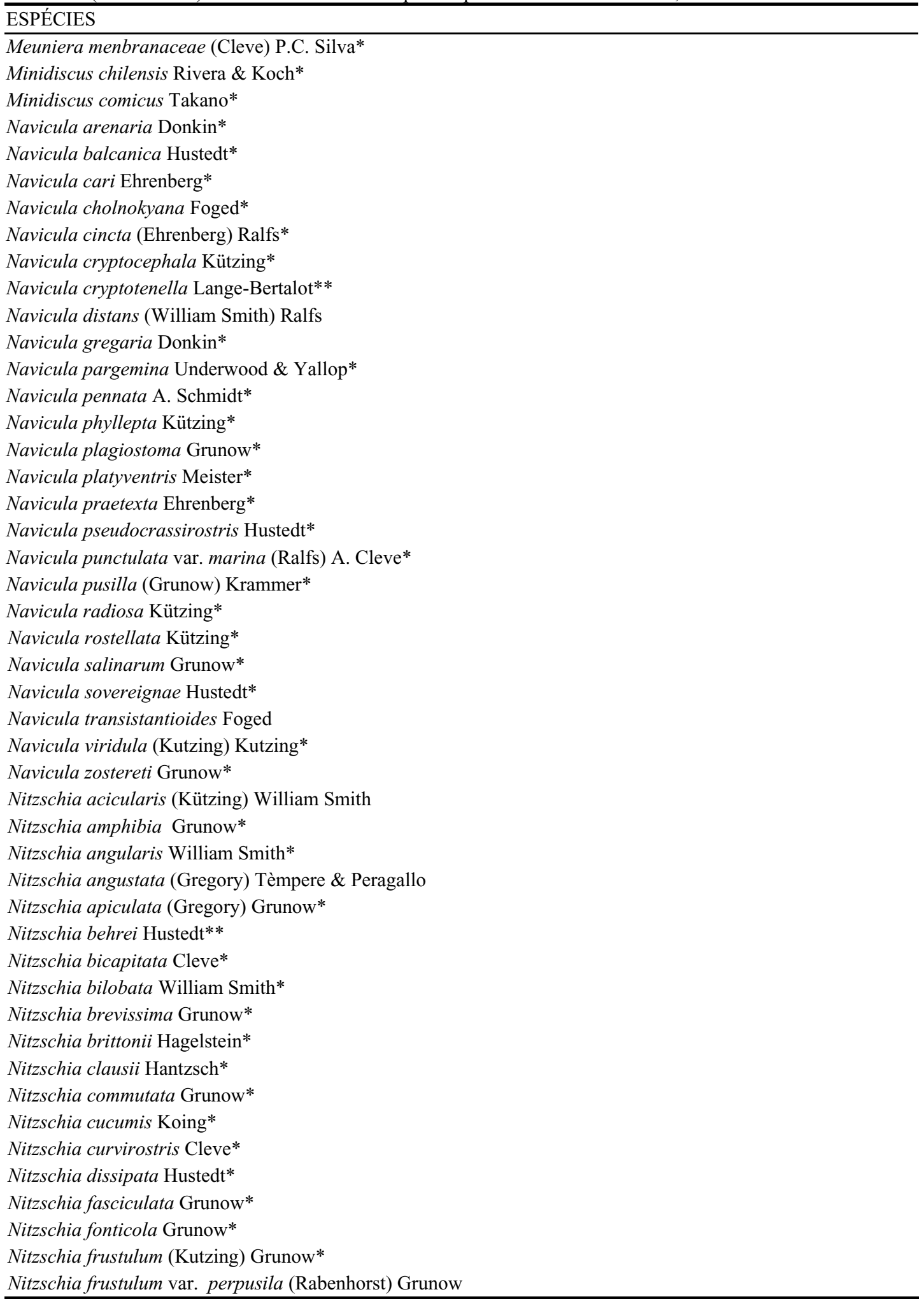

http://www.biotaneotropica.org.br 
TABELA 1 (continuação) - Lista das espécies de diatomáceas citadas para o litoral do Paraná, de 1918 a 2005. TABLE 1 (continuation) - Check-list of diatoms species quoted for the Paraná coast, from 1918 to 2005.

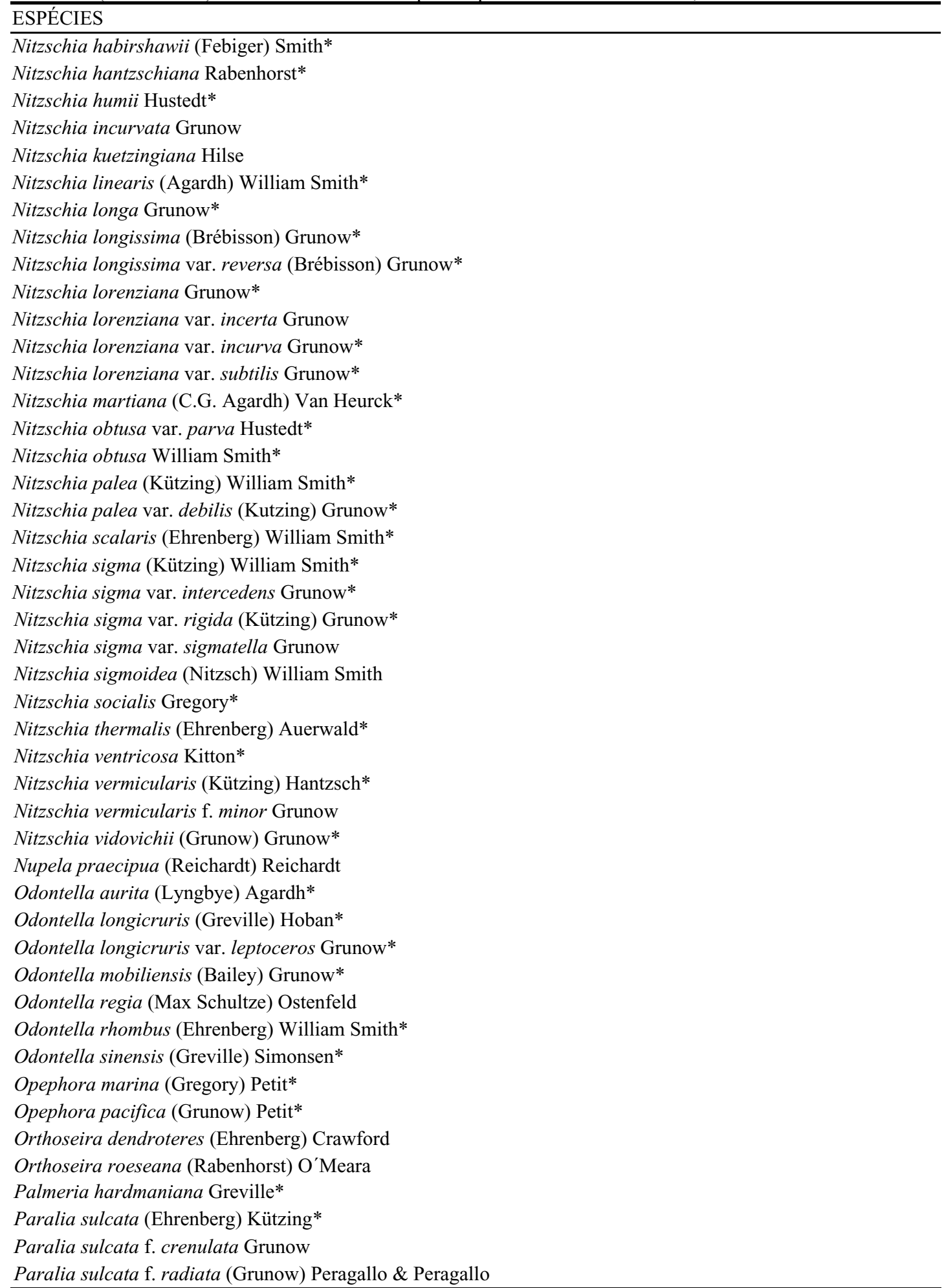

http://www.biotaneotropica.org.br 
TABELA 1 (continuação) - Lista das espécies de diatomáceas citadas para o litoral do Paraná, de 1918 a 2005. TABLE 1 (continuation) - Check-list of diatoms species quoted for the Paraná coast, from 1918 to 2005.

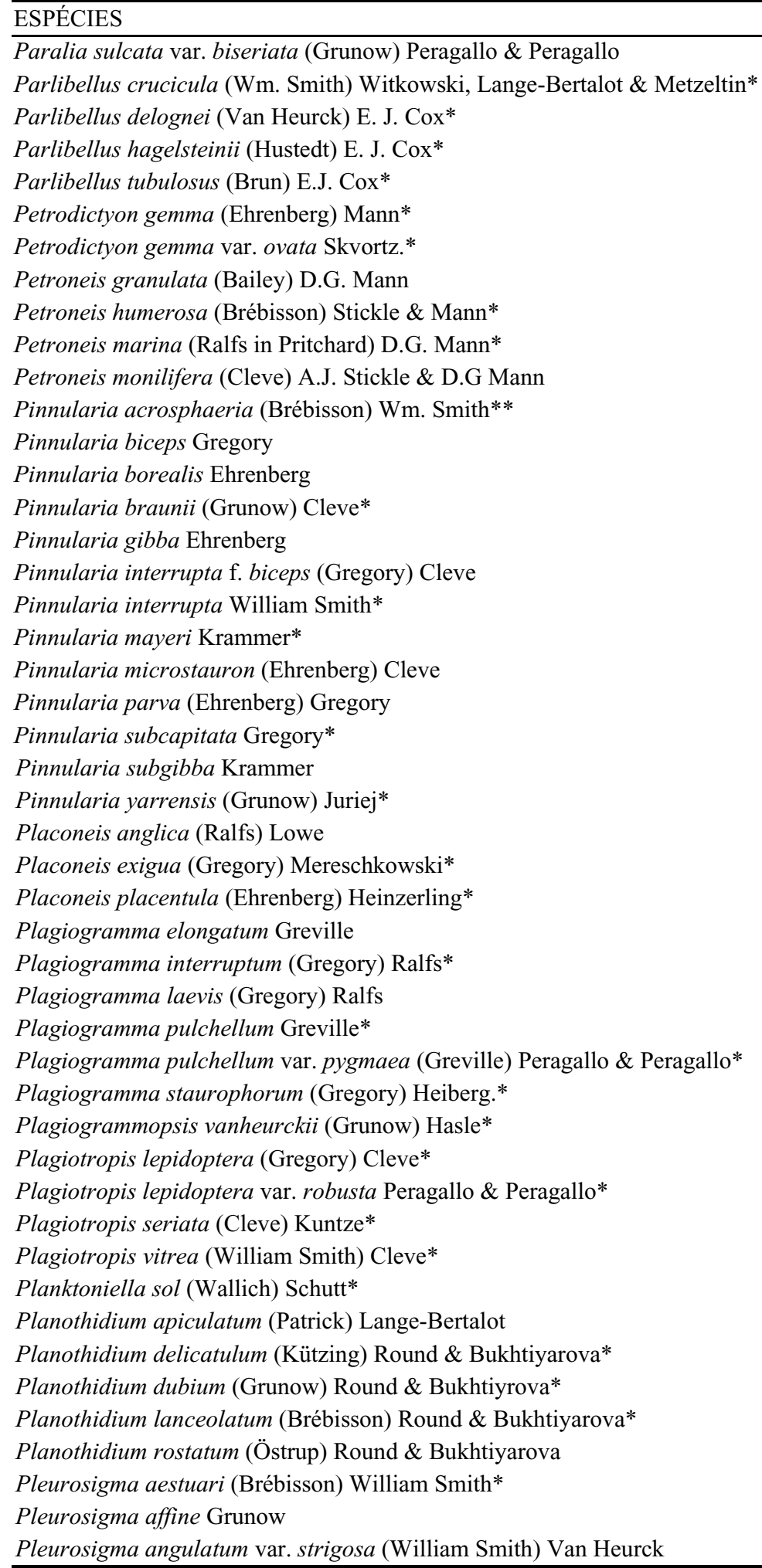

http://www.biotaneotropica.org.br 
TABELA 1 (continuação) - Lista das espécies de diatomáceas citadas para o litoral do Paraná, de 1918 a 2005. TABLE 1 (continuation) - Check-list of diatoms species quoted for the Paraná coast, from 1918 to 2005.

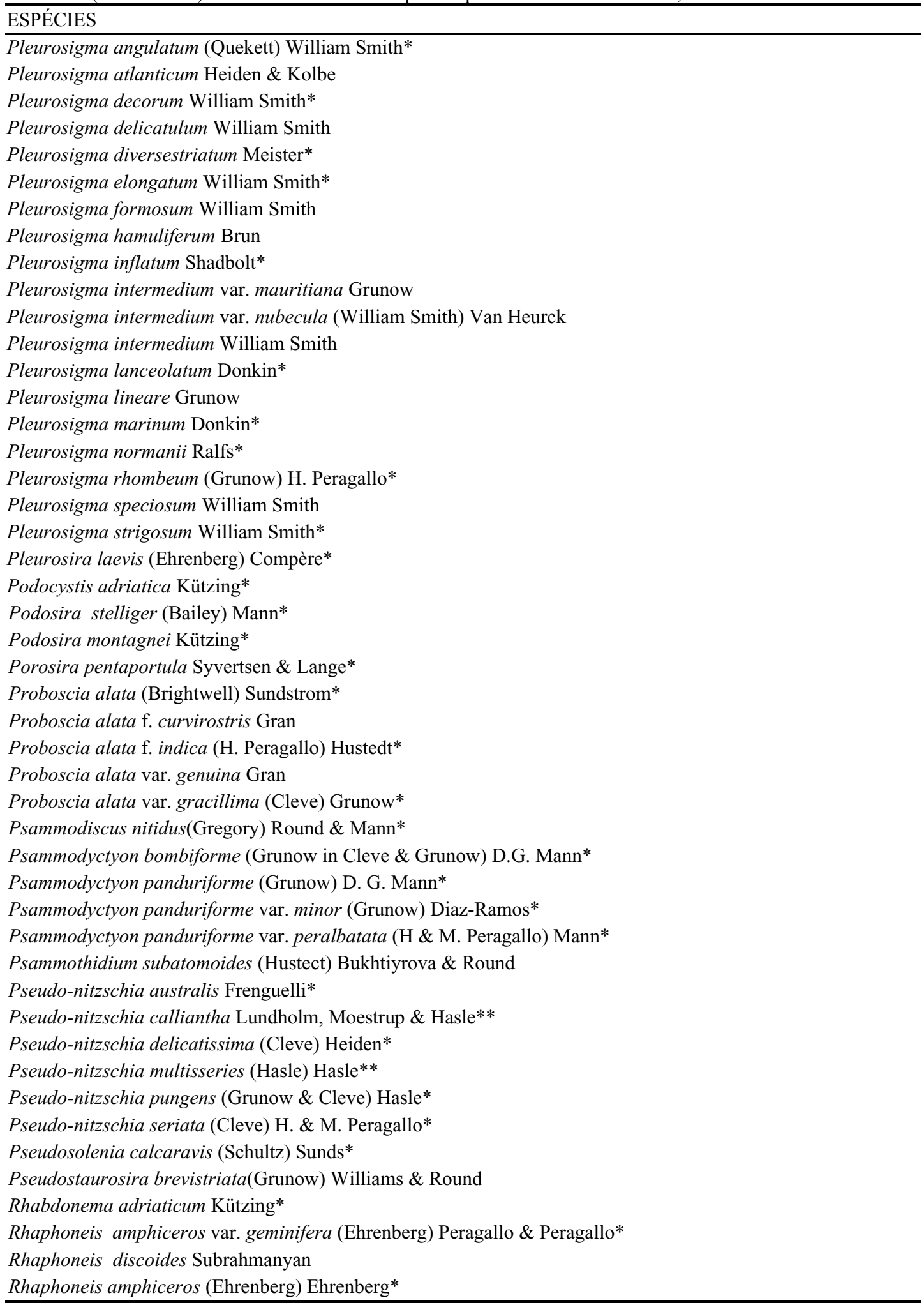

http://www.biotaneotropica.org.br 
TABELA 1 (continuação) - Lista das espécies de diatomáceas citadas para o litoral do Paraná, de 1918 a 2005. TABLE 1 (continuation) - Check-list of diatoms species quoted for the Paraná coast, from 1918 to 2005.

\section{ESPÉCIES}

Pleurosigma angulatum (Quekett) William Smith*

Pleurosigma atlanticum Heiden \& Kolbe

Pleurosigma decorum William Smith*

Pleurosigma delicatulum William Smith

Pleurosigma diversestriatum Meister*

Pleurosigma elongatum William Smith*

Pleurosigma formosum William Smith

Pleurosigma hamuliferum Brun

Pleurosigma inflatum Shadbolt*

Pleurosigma intermedium var. mauritiana Grunow

Pleurosigma intermedium var. nubecula (William Smith) Van Heurck

Pleurosigma intermedium William Smith

Pleurosigma lanceolatum Donkin*

Pleurosigma lineare Grunow

Pleurosigma marinum Donkin*

Pleurosigma normanii Ralfs*

Pleurosigma rhombeum (Grunow) H. Peragallo*

Pleurosigma speciosum William Smith

Pleurosigma strigosum William Smith*

Pleurosira laevis (Ehrenberg) Compère*

Podocystis adriatica Kützing*

Podosira stelliger (Bailey) Mann*

Podosira montagnei Kützing*

Porosira pentaportula Syvertsen \& Lange*

Proboscia alata (Brightwell) Sundstrom*

Proboscia alata f. curvirostris Gran

Proboscia alata f. indica (H. Peragallo) Hustedt*

Proboscia alata var. genuina Gran

Proboscia alata var. gracillima (Cleve) Grunow*

Psammodiscus nitidus(Gregory) Round \& Mann*

Psammodyctyon bombiforme (Grunow in Cleve \& Grunow) D.G. Mann*

Psammodyctyon panduriforme (Grunow) D. G. Mann*

Psammodyctyon panduriforme var. minor (Grunow) Diaz-Ramos*

Psammodyctyon panduriforme var. peralbatata (H \& M. Peragallo) Mann*

Psammothidium subatomoides (Hustect) Bukhtiyrova \& Round

Pseudo-nitzschia australis Frenguelli*

Pseudo-nitzschia calliantha Lundholm, Moestrup \& Hasle**

Pseudo-nitzschia delicatissima (Cleve) Heiden*

Pseudo-nitzschia multisseries (Hasle) Hasle**

Pseudo-nitzschia pungens (Grunow \& Cleve) Hasle*

Pseudo-nitzschia seriata (Cleve) H. \& M. Peragallo*

Pseudosolenia calcaravis (Schultz) Sunds*

Pseudostaurosira brevistriata(Grunow) Williams \& Round

Rhabdonema adriaticum Kützing*

Rhaphoneis amphiceros var. geminifera (Ehrenberg) Peragallo \& Peragallo*

Rhaphoneis discoides Subrahmanyan

Rhaphoneis amphiceros (Ehrenberg) Ehrenberg*

http://www.biotaneotropica.org.br 
TABELA 1 (continuação) - Lista das espécies de diatomáceas citadas para o litoral do Paraná, de 1918 a 2005. TABLE 1 (continuation) - Check-list of diatoms species quoted for the Paraná coast, from 1918 to 2005.

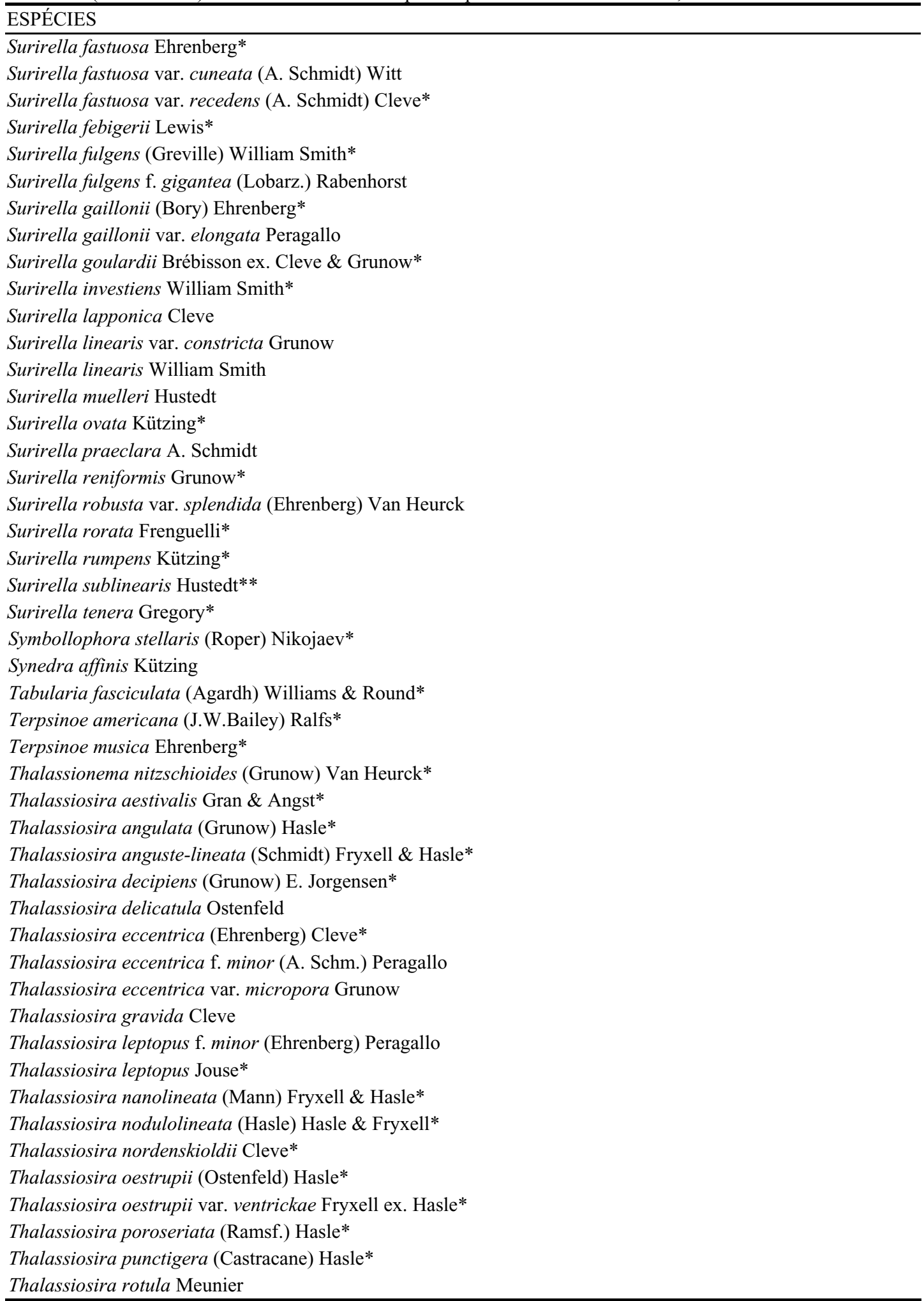

http://www.biotaneotropica.org.br 
TABELA 1 (continuação) - Lista das espécies de diatomáceas citadas para o litoral do Paraná, de 1918 a 2005. TABLE 1 (continuation) - Check-list of diatoms species quoted for the Paraná coast, from 1918 to 2005.

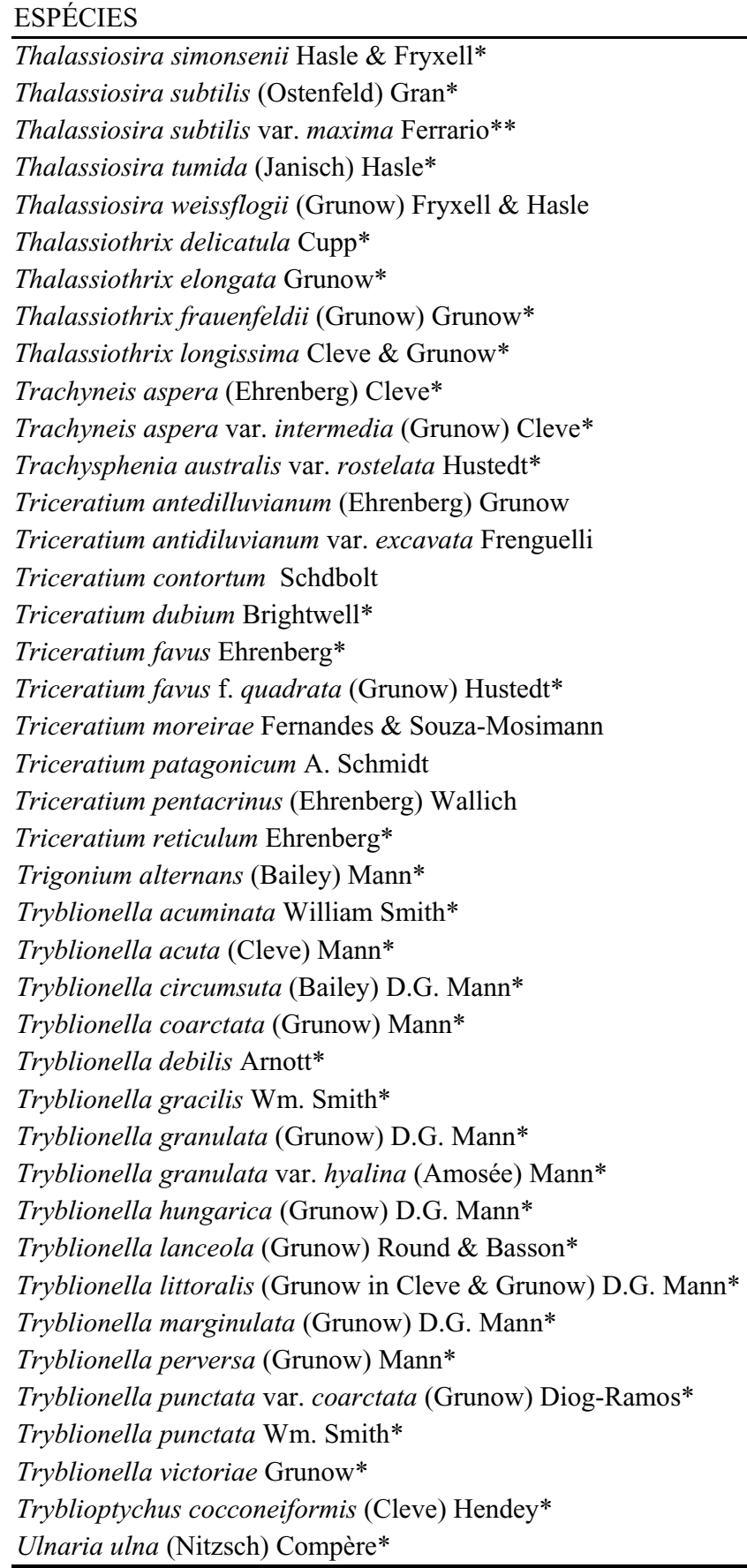

http://www.biotaneotropica.org.br 
TABELA 2 - Espécies de diatomáceas potencialmente nocivas para o litoral do Paraná. TABLE 2 - Diatom species potentially harmful to the Parana State shore.

\begin{tabular}{|c|c|c|c|}
\hline Família & Espécie & nocividade & Referências \\
\hline Skeletonemataceae & Skeletonema costatum & $\begin{array}{l}\text { Injúrias mecânicas em } \\
\text { organismos filtradores, } \\
\text { alteração de cor da água }\end{array}$ & $\begin{array}{l}\text { Fryxell \& Villac (1999), } \\
\text { Méndez \& Ferrari } \\
(2002)\end{array}$ \\
\hline \multirow[t]{3}{*}{ Coscinodiscaceae } & Coscinodiscus centralis & $\begin{array}{l}\text { Injúrias mecânicas em } \\
\text { organismos filtradores. }\end{array}$ & $\begin{array}{l}\text { Fryxell \& Villac (1999), } \\
\text { Ferrario et al. (2002) }\end{array}$ \\
\hline & Coscinodiscus concinnus & $\begin{array}{l}\text { Injúrias mecânicas em } \\
\text { organismos filtradores }\end{array}$ & $\begin{array}{l}\text { Fryxell \& Villac (1999), } \\
\text { Ferrario et al. (2002) }\end{array}$ \\
\hline & Coscinodiscus wailesii & $\begin{array}{l}\text { Injúrias mecânicas em } \\
\text { organismos filtradores, } \\
\text { depleção de oxigênio }\end{array}$ & $\begin{array}{l}\text { Fryxell \& Villac (1999), } \\
\text { Fernandes et al. (2001), } \\
\text { Reguera (2002), Proença } \\
\text { \& Fernandes (2004) }\end{array}$ \\
\hline Hemiaulaceae & Cerataulina pelagica & $\begin{array}{c}\text { Injúrias mecânicas em } \\
\text { organismos filtradores, } \\
\text { anoxia }\end{array}$ & $\begin{array}{l}\text { Hasle \& Fryxell (1995), } \\
\text { Ferrario et al. (2002) }\end{array}$ \\
\hline \multirow[t]{3}{*}{ Chaetocerataceae } & Chaetoceros concavicornis & $\begin{array}{l}\text { Injúrias mecânicas em } \\
\text { organismos filtradores }\end{array}$ & $\begin{array}{l}\text { Fryxell \& Villac (1999), } \\
\text { Reguera (2002) }\end{array}$ \\
\hline & Chaetoceros convolutus & $\begin{array}{l}\text { Injúrias mecânicas em } \\
\text { organismos filtradores }\end{array}$ & Fryxell \& Villac (1999) \\
\hline & Chaetoceros danicus & $\begin{array}{l}\text { Injúrias mecânicas em } \\
\text { organismos filtradores }\end{array}$ & Ferrario et al. (2002) \\
\hline \multirow[t]{2}{*}{ Leptocylindraceae } & Leptocylindrus danicus & $\begin{array}{l}\text { Injúrias mecânicas em } \\
\text { organismos filtradores }\end{array}$ & Fryxell \& Villac (1999) \\
\hline & Leptocylindrus minimus & $\begin{array}{l}\text { Injúrias mecânicas em } \\
\text { organismos filtradores }\end{array}$ & $\begin{array}{l}\text { Fryxell \& Villac (1999), } \\
\text { Reguera (2002), Rivera } \\
\text { et al. (2002) }\end{array}$ \\
\hline Fragilariaceae & Asterionellopsis glacialis & $\begin{array}{l}\text { Injúrias mecânicas em } \\
\text { organismos filtradores }\end{array}$ & Odebrecht et al. (2002) \\
\hline Catenulaceae & Amphora coffaeformis & Potencialmente tóxica & $\begin{array}{l}\text { Sala et al. (1998), } \\
\text { Ferrario et al. (2002) }\end{array}$ \\
\hline \multirow[t]{6}{*}{ Bacillariaceae } & Pseudo-nitzschia australis & Potencialmente tóxica & $\begin{array}{l}\text { Fryxell \& Villac (1999), } \\
\text { Reguera (2002), Ferrario } \\
\text { et al. (2002), Odebrecht } \\
\text { et al. (2002) }\end{array}$ \\
\hline & Pseudo-nitzschia calliantha & Potencialmente tóxica & Lundholm et al. (2003) \\
\hline & Pseudo-nitzschia delicatissima & Potencialmente tóxica & $\begin{array}{l}\text { Fryxell \& Villac (1999), } \\
\text { Ferrario et al. (2002) }\end{array}$ \\
\hline & Pseudo-nitzschia multisseries & Potencialmente tóxica & $\begin{array}{l}\text { Fryxell \& Villac (1999), } \\
\text { Ferrario et al. (2002), } \\
\text { Odebrecht et al. (2002) }\end{array}$ \\
\hline & Pseudo-nitzschia pungens & Potencialmente tóxica & $\begin{array}{l}\text { Fryxell \& Villac (1999), } \\
\text { Ferrario et al. (2002), } \\
\text { Odebrecht et al. (2002) }\end{array}$ \\
\hline & Cylindrotheca closterium & $\begin{array}{l}\text { Injúrias mecânicas em } \\
\text { organismos filtradores }\end{array}$ & $\begin{array}{l}\text { Fanuko et al. (1989), } \\
\text { Stachowitsch et al. } \\
(1990)\end{array}$ \\
\hline
\end{tabular}




\section{Referências Bibliográficas}

BATES, S.S.,BIRD, C.J., DE FREITAS, A.S.W., FOXALL, R., GILGAN, M., HANIC, L.A., JOHNSON, G.R., McGULLOCH,A.W., ODENSE, P., POCKLINGTON, R., QUILLIAM, M.A., SIM, J.C., SUBBA RAO, E.C.D., TOOD, J.A., WALTER. \& WRIGHT, J.L.C. 1989. Pennate diatom Nitzschia pungens as the primary source of domoic acid, a toxin in shellfish from eastern Prince Edward Island, Canadá. Can. J. Fish. Aquat. Sci. 46:1203-1215.

BATES, S.S., GARRISON, D.L., HORNER, R.A. 1998. Bloom dynamics and physiology of domoic acid producing Pseudo-nitzschia species. In Physiological ecology of harmful algal blooms (D.M Anderson, A.D. Cembella \& G.M. Hallegraef, eds), Spring - Verlag, New York, p.267-292.

BRANDINI, F.P. 1985a. Seasonal succession of the phytoplankton in the bay of Paranaguá (Paraná State - Brazil). Rev. Bras. Biol. 45: 687-694.

BRANDINI, F.P. 1985b. Ecological studies in the bay of Paranaguá. I. Horizontal distribution and seasonal dynamics of the phytoplankton. Bol. Inst. Oceanogr. 33: $139-147$.

BRANDINI, F.P. \& MORAES, C.B. 1986. Composição e distribuição do fitoplâncton em áreas costeiras e oceânicas da região sueste do Brasil. Neritica 1(3):9-19.

BRANDINI, F.P. 1988 a. Composição e distribuição do fitoplâncton na região Sueste do Brasil e suas relações com as massas de água (Operação Sueste- Julho/Agosto 1982). Cienc. Cult. 40(4): 334-341.

BRANDINI, F.P. 1988 b. Hydrography, phytoplankton biomass and photosynthesis in shelf and oceanic waters off Southeastern Brazil during autumn (May/June, 1983). Bol. Inst. Oceanogr. 36(1/2):63-72.

BRANDINI, F.P., MORAES, C.L.B. \& THAMM, C.A.C. 1989. Shelf-break upwelling, subsurface maxima of chlorophyll and nitrite, and vertical distribution of a subtropical nano-microplankton community off southeastern Brazil. In Memórias do III Encontro Brasileiro de Plâncton (F.P Brandini, coord.), Curitiba, p.47-55.

BRANDINI, F. P. 1990a. Hydrography and characteristics of the phytoplankton in shelf and oceanic waters off southeastern Brazil during winter (July/August 1982) and summer (February/ March 1984). Hidrobiologia 196:111-148.

BRANDINI, F.P. 1990b. Produção primária e características fotossintéticas do fitoplâncton na região sueste do Brasil. Bol. Inst. Oceanogr. 38(2):147-159.

BRANDINI, F.P. \& THAMM, C. AC. 1994. Variações diárias e sazonais do fitoplâncton e parâmetros ambientais na baía de Paranaguá. Neritica 8(1-2):55-72.
BRANDINI, F.P. \& FERNANDES, L.F. 1996. Microalgae of the continental shelf off Paraná state, southeastern Brazil: a review of studies. Rev. Bras. Oceanogr. 44(1):69-80.

BRANDINI, F.P., LOPES, R.M., GUTSEIT, K. S., SPACH, H.L. \& SASSI, R. 1997. Planctonologia na plataforma continental do Brasil - Diagnose e revisão bibliográfica. FEMAR, Rio de Janeiro.

BRANDINI, F.P., SILVA, E.T., PELIZZARI, F.M., FONSECA, A.L.O. \& FERNANDES, L.F. 2001. Production and biomass accumulation of periphytic diatoms growing on glass slides during a 1-year cycle in a subtropical estuarine environment (Bay of Paranaguá, southern Brazil). Mar. Biol. 138:163-171.

CUNHA, A.M. \& FONSECA, O. 1918. O microplancton das costas meridionais do Brasil. Memórias do Instituto Oswaldo Cruz. 10(2):99-103.

DANIELIDIS, D.B. \& MANN, D.G. 2002. The systematics of Seminavis (Bacillariophyta): the lost identities of Amphora angusta, A. ventricosa and A. macilenta. Europ. J. Phycol. 37:429-448.

FANUKO, N., RODE, J \& DRASLAR, K. 1989. Microflora from the Adriatic mucous aggregations. Biol. Vestn. 4:27-34.

FEHLING, J., GREEN, D.H., DAVIDSON, K., BOLCH, C.J. \& BATES, S.S. 2004. Domoic acid production by Pseudonitzschia seriata (Bacillariophyceae) in Scottish waters. J. Phycol. 40(4):622-630.

FERNANDES, L.F. 2003. New observations on frustule morphology of Eupodiscus radiatus Bailey and Fryxelliella floridana Prasad. Braz. J. Biol. 63(3):411-421.

FERNANDES, L.F., BRANDINI, F.P., GUTSEIT, K.S., FONSECA, A.L. \& PELIZZARI, F.M. 1999. Benthic diatoms growing on glass slides in the Paranaguá Bay, Southern Brazil: taxonomic structure and seasonal variation. Insula 28: 53-100.

FERNANDES, L.F. \& SOUZA-MOSIMANN, R.M. 2001. Triceratium sp. nov. and Triceratium dubium (Triceratiaceae-Bacillariophyta) from estuarine environments of Southern Brazil, with comments on the genus Triceratium C.G. Ehrenberg. Rev. Bras. Biol. 61(11):159-170.

FERNANDES, L. F., ZEHNDER-ALVES, L. \& BASSFELD, J. 2001. The recently established diatom Coscinodiscus wailesii (Coscinodiscales, Bacillariophyta) in Brazilian waters. I: remarks on morphology and distribution. Phycol. Res. 49:89-96.

FERNANDES, L.F., ESKINAZI-LEÇA, E., MOURA,A.N. \& OLIVEIRA, G.G. 2002. The occorrence of Nitzschia martiana (C.A. Agardh) Van Heurck (NitzschiaceaeBacillariophyta) in the southwestern Atlantic Ocean. Hoehnea. 29(2):119-131. 
FERNANDES, L.F. \& BRANDINI, F.P. 2004. Diatom associations in shelf waters off Parana State, Southern Brazil: annual variation in relation to environmental factors. Braz. J. Oceanogr. 52(1):19-34.

FERRARIO, M., SAR, E. \& SALA, S. 2002. Diatomeas potencialmente toxígenas del cono Sur Americano. In Floraciones algales nocivas en el Cono Sur Americano (E.A. Sar, M.E. Ferrario, \& B. Reguera, eds). Instituto Español de Oceanografia, p. 169-194.

FRYXELL, G.R. \& VILLAC, M.C. 1999. Toxic and harmful marine diatoms. In The diatoms: applications for the environmental and earth sciences (E. F. Stoemer \& J. P. Smol, eds.). Cambridge University Press, Cambridge, p.1-469.

GLOBAL BALLAST WATER MANAGEMENT PROGRAMME. The GLOBALLAST Programme: http://

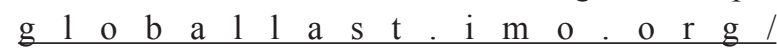
index.asp?page $=$ gef interw project.htm\&menu $=$ true (último acesso em 12/01/2006).

HÅKANSON, H.A. 2002. A compliation and evaluation of species in the general Stepahnodiscus, Cyclostephanos and Cyclotela with a new genus in the family Stephanodiscaceae. Diatom Res. 17(1):1-139.

HASLE, G.R. 2002. Are most of the domoic acid-producing species of the diatom genus Pseudo-nitzschia cosmopolites? Harmful Algae 1:137-146.

HASLE, G. R. \& FRYXELL, G. A. 1970. Diatoms: cleaning and mouthing for ligth and electron microscope. Trans. Am. Microsc. Soc. 89:469-474.

HASLE, G.R. \& FRYXELL, G.A. 1995. Taxonomy of diatoms. In Manual on harmful marine microalgae (G.M. Hallegraeff, D.M. Anderson \& A.D. Cembella, eds). Ioc Manuals and Guides, p.1-22.

HASLE, G.R \& SYVERTSEN, E.E. 1997. Marine diatoms. In Identifying Marine Phytoplankton (C.R. Tomas, ed.). Academic Press, London, p. 45 -88.

HASLE, G.R., LANGE, C.B. \& SYVERTSEN, E.E. 1996. Areview of Pseudo-nitzschia, with reference to the Skagerrak, north atlantic, and adjacent waters. Hegol. Meeresunlers. 50:131-175.

INTERNATIONAL OCEANOGRAPHIC COMMISSION. 2005. The IOC harmful algal bloom programme: http:// ioc.unesco.org/hab (último acesso em 30/06/2005).

LUNDHOLM, N., MOESTRUP, Ø., HASLE, G.R. \& HOEFEMDEN, K. 2003. A study of the Pseudo-nitzschia pseudodelicatissima/cuspidata complex (Bacillariophyceae): what is P. pseudodelicatissima? J. Phycol. 39:797-813.
MARTINS, G.J., MARONE, E., ANGULO, R.J., NOERNBERG, M.A. \& QUADROS, C.J.L. 2004. Dinâmica da zona rasa de shoaling e o transporte de sedimentos na desembocadura sul do Complexo Estuarino de Paranaguá -PR. Bol. Paraná. Geocienc. 54: 51 - 64.

MÉNDEZ, S \& FERRARI, G. 2002. Floraciones algales nocivas em Uruguai: antecedents, proyectos en curso y revisíon de resultados In Floraciones algales nocivas en el Cono Sur Americano (E.A. Sar, M.E. Ferrario \& B Reguera, eds). Instituto Español de Oceanografia, p.271-288.

MOREIRA FILHO, H. 1959. Diatomáceas do Paraná: I. A flora diatomológica no Sargassum. Bol. Inst. Hist. Nat., Bot. 2:1-18.

MOREIRA FILHO, H. 1960. Diatomáceas do trato digestivo de Tegula viridula Gmeelin. Bol. Univ. Fed. Parana, Bot. 1:1-24.

MOREIRA FILHO, H. 1961. Diatomáceas da Baía de Guaratuba. Bol. Univ. Fed. Parana, Bot. 3:1-35.

MOREIRA FILHO, H \& KUTNER, M. B. 1962. Contribuição para o conhecimento das diatomáceas do Manguezal de Alexandra. Bol. Univ. Fed. Parana, Bot. 4:1-24.

MOREIRA FILHO, H. \& MÔMOLI, D. M. 1962. Sobre a presença de diatomáceas em alguns sambaquis do litoral paranaense. Bol. Univ. Fed. Parana, Bot. 5:1-9.

MOREIRA-FILHO, H. \& MÔMOLI, D.M. 1966. Diatomáceas em alguns focos larvários de anofelinos de curitiba (Paraná-Brasil). Bol. Univ. Fed. Parana, Bot. 15:1-6.

MOREIRA-FILHO, H. 1968. Margaritum (Podosira) tenebro (Leuduger-Fortmorel) nov. Genus et nova comb. Bol. Univ. Fed. Parana, Bot. 20:1-4.

MOREIRA-FILHO, H., VALENTE-MOREIRA, I. M. \& CECY, I.I.T. 1975. Diatomáceas da Baía de Paranaguá, Estado do Paraná, Brasil (Chrysophyta-Bacillariophyceae). Bol. Mus. Bot. Munic. 20:1-25.

MOREIRA-FILHO, H.; VALENTE-MOREIRA, I.M. \& MATOS, A. 1977. Diatomáceas epífitas em Codium decorticatum (Wood.) Howe. Trib. Farm. 44(6):3-17.

MOREIRA-FILHO, H. \& VALENTE-MOREIRA, I.M. 1979. Diatomáceas da enseada da Prainha, Matinhos, Paraná. Bol. Mus. Bot. Munic. 35:1-12.

MOREIRA-FILHO, H. \& VALENTE-MOREIRA, I. M. 1980. Diatomáceas epífitas em Ulva fasciata Delile. Bol. Mus. Bot. Munic. 41:1-10.

MOREIRA-FILHO, H. \& VALENTE-MOREIRA, I. M. 1981. Avaliação taxonômica e ecológica das diatomáceas (Bacillariophyceae) epífitas em algas pluricelulares obtidas nos litorais dos estados do Paraná, Santa Catarina e São Paulo. Bol. Mus. Bot. Munic. 47:1-17.

MOREIRA-FILHO, H. \& VALENTE-MOREIRA, I.M. 1984. Catálogo das diatomáceas (ChrysophytaBacillariophyceae) marinhas e estuarinas do Estado do Paraná, Brasil. Acta Biol. Parana. 13(1, 2, 3, 4):3-49. 
MOREIRA-FILHOH., VALENTE-MOREIRAI.M., SOUZAMOSIMANN R.M, \& CUNHA J.A. 1990. Avaliação florística e ecológica das diatomáceas (ChrysophytaBacillariophyceae) marinhas e estuarinas nos estados do Paraná, Santa Catarina e Rio Grande do Sul. Estud. Biol. 25:5-48.

ODEBRECHT, C., AZEVEDO, S.M.F.O., GARCIA, V.M.T., HUSZAR, V.L.M., MAGALHÃES, V.F., MENEZES, M., PROENÇA, L.A.O., RÖRIG, L.R., TENENBAUM, D.R., VILLAC, M.C. \& YUNES, J.S. 2002. Floraciones de microalgas nocivas en Brasil: estado del arte y proyectos en curso. In In Floraciones algales nocivas en el Cono Sur Americano (E.A. Sar, M.E. Ferrario \& B Reguera, eds). Instituto Español de Oceanografia, p.217-233.

OLIVEIRA, R.J.M. 1983. Um levantamento quantitativo relativo do gênero Actinoptychus Ehrenberg (Chrysophyta-Bacillariophyceae) no estuário do Rio Itiberê, Paranaguá, Paraná, Brasil. Estud. Biol. 8:1-27.

PROENÇA, L.A.O. \& FERNANDES, L.F. 2004. Introdução de microalgas no ambiente marinho: impactos negativos e fatores controladores. In Água de lastro e bioinvasão (J. S. V. Silva \& R.C.C.L Souza, eds). Editora Interciência, Rio de Janeiro, p.1-224.

REGUERA, B. 2002. Estabelecimiento de um programa de seguimiento de microalgas toxicas. In In Floraciones algales nocivas en el Cono Sur Americano (E.A. Sar, M.E. Ferrario, \& B Reguera, eds). Instituto Español de Oceanografia, p.21-54.

RINES, J.E.B.; DONAGHAY, P.L.; DESKSHENIEKS, M.M. \& SULLIVAN, J.M. 2002. Thin layers and camouflage: hidden Pseudo-ntzschia spp. (Bacillariophyceae) populations in a fjord in the San Juan Islands, Washington, USA. Mar. Ecol. Ser. (225):123-137.

RIVERA, P., CRUCES, F. \& CLEMENTE, A. 2002. Leptocylindrus minimus (Bacillariophyceae): morfologia y distribucion in Chile. Gayana, Bot. 59(1):7-11.

ROUND, F.E. \& BUKHTIYAROVA, L. 1996. Four new genera based on Achnanthes (Achnantidium) together with a redefinition of Achnanthidium. Diatom Res. 11(2):345-361.

SALA, S.E., SAR, E.A. \& FERRARIO, M.E. 1998. Rewiew of material reported as containing Amphora coffaeformis (Agardh) Kützing in Argentina. Diatom Res. 13(2):223-336.

SOUZA-MOSIMANN R.M., FERNANDES L.F. \& LUDWIG, T.A.V. 1997. The estuarine benthic diatom Margaritum terebro (Bacillariophyta, Hyalodiscaceae): morphology and taxonomy. Rev. Bras. Oceanogr. 45(1/2):45-52.

STANCHOWITSCH, M. FANUKO, N. \& RICHTER, M. 1990. Mucus aggregates in the Adriatic Sea: an overviwe of stages and occurrences. Mar. Ecol. 11:327-350.

TAVARES, B \& VALENTE-MOREIRA, I.M. 2000. Diatomoflórula do Lago de Cascavel, Município de Cascavel, Estado do Paraná, Brasil. Hoehnea 27(1):1-24.
VALENTE-MOREIRA, I. M.; MOREIRA-FILHO, H.; CUNHA, J. A. \& LUDWIG, T. A. V. 1980. Diatomáceas epífitas em Padina vickersiae Hoyt ex Howe. Trib. Farm. 48(1-2):114-122.

VALENTE-MOREIRA, I.M. \& MOREIRA FILHO, H. 1981. Diatomáceas de Pontal do Sul, Município de Paranaguá, Estado do Paraná, Brasil. (Chrysophyta, Bacillariophyceae). Phycol. Lat. Amer. Vaduz. 52:156-185.

VALENTE-MOREIRA, I.M. \& MOREIRA FILHO, H. 1982. Contribuição ao estudo das Bacillariophyceae (Diatomáceas) de Caiobá, Estado do Paraná, Brasil. Acta Biol. Parana. 110(11):157-197.

VALENTE-MOREIRA, I.M., MOREIRA-FILHO, H. \& CUNHA, J.A. 1985. Diatomáceas (Bacillariophyceae) marinhas e estuarinas nas praias de Atami e Shangri-lá, município de Paranaguá, estado do Paraná, Brasil. Estud. Biol. 12:1-47.

VALENTE-MOREIRA, I.M., MOREIRA-FILHO, H. \& CUNHA, J.A. 1986. Diatomáceas (Chrysophyta, Bacillariophyceae) marinhas e estuarinas no canal da Galheta, Baía de Paranaguá, Paraná, Brasil. Acta Biol. Parana. 19(1-2-3-4):21-44.

VALENTE-MOREIRA, I.M., MOREIRA-FILHO, H.; CUNHA, J.A. \& SHIRATA, M.T. 1987. A flórula diatomológica marinha e estuarina nos balneários de Canoas e Ipanema, estado do Paraná, Brasil. I. Estudo qualitativo e ecológico. Estud. Biol. 17:23-48.

VALENTE-MOREIRA,I.M., MOREIRA-FILHO,H.\&CUNHA, J.A. 1994a. Diatomáceas (Chrysophyta, Bacillariophyceae) em biótopo do manguezal do rio Perequê, em Pontal do Sul, Paranaguá, Estado do Paraná, Estado do Paraná, Brasil. Acta Biol. Parana. 23(1, 2, 3, 4): 55-72.

VALENTE-MOREIRA, I.M., MOREIRA-FILHO,H., CUNHA, J.A. \& NAIKAMURA, I.T. 1994b. Diatomáceas (Chrysophyta - Bacillariophyceae) no conteúdo estomacal de peixes e crustáceos do Manguezal do Rio Perequê, Pontal do Sul, Estado do Paraná, Brasil. Estud. Biol. 03(39):99-114.

Título: Diatomáceas Marinhas e Estuarinas do Paraná

Autores: Procopiak, L.K., Fernandes, L.F. and Moreira Filho, $\mathrm{H}$.

Biota Neotropica, Vol.6 ( número 3): 2006

http://www.biotaneotropica.org.br/v6n3/pt/ abstract?inventory+bn02306032006

Recebido em 08/03/06 - Versão reformulada recebida em 04/09/06 - Publicado em 11/10/06

ISSN 1676-0603 